\title{
Galactic Bulge microlensing optical depth from EROS-2 ${ }^{\star \star \star}$
}

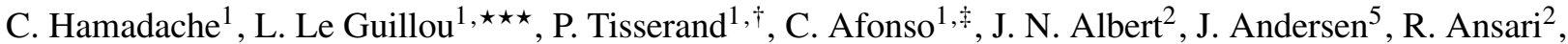 \\ É. Aubourg ${ }^{1}$, P. Bareyre ${ }^{1}$, J. P. Beaulieu ${ }^{3}$, X. Charlot ${ }^{1}$, C. Coutures ${ }^{1,3}$, R. Ferlet ${ }^{3}$, P. Fouqué ${ }^{7,8}$, J. F. Glicenstein ${ }^{1}$, \\ B. Goldman ${ }^{1, \ddagger}$, A. Gould ${ }^{6}$, D. Graff ${ }^{6, \S}$, M. Gros ${ }^{1}$, J. Haissinski ${ }^{2}$, J. de Kat ${ }^{1}$, É. Lesquoy ${ }^{1,3}$, C. Loup ${ }^{3}$, C. Magneville ${ }^{1}$, \\ J. B. Marquette ${ }^{3}$, É. Maurice ${ }^{4}$, A. Maury ${ }^{8, \mathbb{I}}$, A. Milsztajn ${ }^{1}$, M. Moniez ${ }^{2}$, N. Palanque-Delabrouille ${ }^{1}$, O. Perdereau ${ }^{2}$, \\ Y. R. Rahal ${ }^{2}$, J. Rich ${ }^{1}$, M. Spiro ${ }^{1}$, A. Vidal-Madjar ${ }^{3}$, L. Vigroux ${ }^{1,3}$, and S. Zylberajch ${ }^{1}$
} (The EROS-2 collaboration)

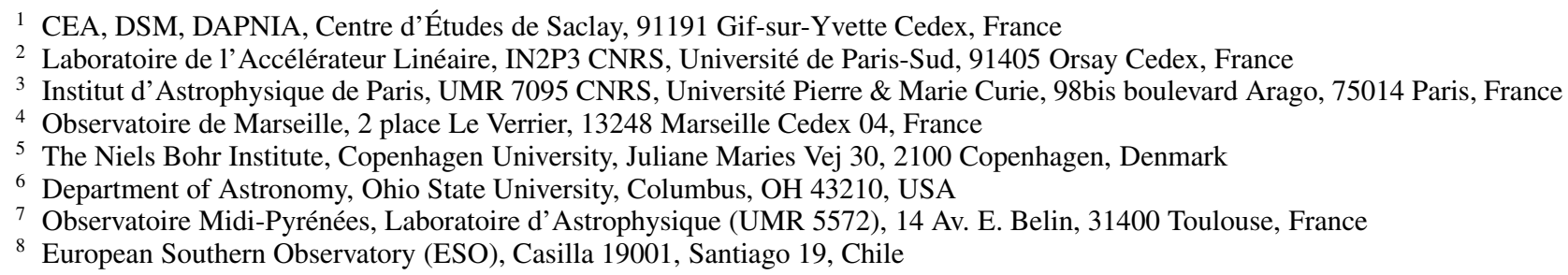

Received 23 January 2006 / Accepted 10 April 2006

\section{ABSTRACT}

\begin{abstract}
Aims. We present a new EROS-2 measurement of the microlensing optical depth toward the Galactic Bulge.
Methods. Light curves of $5.6 \times 10^{6}$ clump-giant stars distributed over $66 \mathrm{deg}^{2}$ of the Bulge were monitored during seven Bulge seasons. 120 events were found with apparent amplifications greater than 1.6 and Einstein radius crossing times in the range $5 \mathrm{~d}<t_{\mathrm{E}}<400 \mathrm{~d}$. This is the largest existing sample of clump-giant events and the first to include northern Galactic fields.

Results. In the Galactic latitude range $1.4^{\circ}<|b|<7.0^{\circ}$, we find $\tau / 10^{-6}=(1.62 \pm 0.23) \exp [-a(|b|-3 \mathrm{deg})]$ with $a=(0.43 \pm$ $0.16) \mathrm{deg}^{-1}$. These results are in good agreement with our previous measurement, with recent measurements of the MACHO and OGLE-II groups, and with predictions of Bulge models.
\end{abstract}

Key words. Galaxy: bulge - Galaxy: stellar contents - Galaxy: structure - gravitational lensing

\section{Introduction}

Gravitational microlensing of stars is an important tool for constraining the quantity and characteristics of faint compact objects between the stars and the observer. In microlensing events, the lensing object passes near the line of sight towards the background star, causing a transient magnification of the star's primary image as well as creating a secondary image. At Galactic scales, neither the image separation nor the image size are normally resolvable, so the only easily observable effect during a

* Based on observations made with the MARLY telescope at the European Southern Observatory, La Silla, Chile.

$\star \star$ Table 3 is only available in electronic form at http://www. edpsciences.org

$\star \star \star$ Now at Instituut voor Sterrenkunde, Celestijnenlaan 200 B, 3001 Leuven, Belgium.

Now at Research School of Astronomy and Astrophysics, Australian National University, Mount Stromlo Obs., Cotter Rd., Weston, ACT 2611, Australia.

$\doteqdot$ Now at Max-Planck-Institut für Astronomie, Koenigstuhl 17, 69117 Heidelberg, Germany.

$\S$ Now at Division of Medical Imaging Physics, Johns Hopkins University, Baltimore, MD 21287-0859, USA.

II Now at San Pedro de Atacama Celestial Exploration, Casilla 21, San Pedro de Atacama, Chile. microlensing event is an apparent transient amplification of the star's flux. The characteristic timescale giving the effective duration of the amplification is proportional to the square root of the lens mass and inversely proportional to the transverse velocity. For Galactic stellar lenses, the timescales are on the order of a few weeks.

Microlensing searches were originally proposed (Paczyński 1986) as a tool for detecting dark matter in galactic halos. Searches for the lensing of stars in the Magellanic Clouds by the MACHO (Alcock et al. 2000b) and EROS-2 (Afonso et al. 2003a; Tisserand et al. 2006) projects have placed constraints on the fraction of the Milky Way halo that can be comprised of faint compact objects. Searches for objects in the halo of M 31 are also being performed with candidate events reported by the VATTColumbia (Uglesich et al. 2004), WeCAPP (Riffeser et al. 2003), AGAPE (Calchi Novati et al. 2005), MEGA (de Jong et al. 2006) and Nainital (Joshi et al. 2005) collaborations. The AGAPE and MEGA collaborations presented efficiency calculations allowing them to constrain the content of the M 31 and Milky Way halos.

Microlensing has also been used as a tool to investigate compact objects in the visible regions of the Milky Way. Besides the Galactic Bulge that is the subject of this paper, microlensing of stars in the spiral arms of the Galaxy have also been studied (Derue et al. 2001). 
Microlensing surveys toward the Galactic Bulge were first proposed (Paczyński 1991; Griest 1991) as a probe of ordinary stars in the Galactic Disk, though it was soon realized (Kiraga \& Paczyński 1994) that lensing by stars in the Bulge itself is of comparable importance. The optical depth, i.e. the probability that at a given time a star at a distance $D_{\mathrm{s}}$ is amplified by more than a factor 1.34 is

$\tau=\frac{4 \pi G D_{\mathrm{S}}^{2}}{c^{2}} \int_{0}^{1} \rho(x) x(1-x) \mathrm{d} x$,

where $\rho$ is the mass density of lenses and $x=D_{\mathrm{l}} / D_{\mathrm{s}}$ is the ratio between the lens and source distances. Very qualitatively, the integral for the Disk contribution to the optical depth is

$\tau_{\text {disk }} \sim \frac{G M_{\text {disk }}}{c^{2} h_{\text {disk }}}$

where $M_{\text {disk }}$ and $h_{\text {disk }}$ are the total mass and scale height of the Disk lensing population. Lenses in the Bulge are near the source star so the Bulge contribution is

$\tau_{\text {bulge }} \sim \frac{G M_{\text {bulge }}}{c^{2} R_{\text {bulge }}} \sim \frac{G \rho_{\text {bulge }} R_{\text {bulge }}^{2}}{c^{2}}$,

where $M_{\text {bulge }}, \rho_{\text {bulge }}$ and $R_{\text {bulge }}$ are the mass, density and line of sight thickness of the Bulge lensing population. For Bulge stars near the direction of the Baade window $\left(\ell=1^{\circ}, b=-3.9^{\circ}\right)$, the most recent calculations (Evans \& Belokurov 2002; Bissantz \& Gerhard 2002; Han \& Gould 2003; Wood \& Mao 2005) give total optical depths in the range $1<\tau / 10^{-6}<2$, with about $60 \%$ of the rate being due to lensing by Bulge stars and the remainder by Disk stars. The expected optical depth naturally has a strong dependence on the Galactic latitude, $b$, falling typically from $\tau \sim$ $5 \times 10^{-6}$ at $\left(l \sim 0,|b|=1^{\circ}\right)$ to $\tau \sim 5 \times 10^{-7}$ at $\left(l \sim 0,|b|=6^{\circ}\right)$.

The first measurements of the optical depth towards the Galactic Bulge by the OGLE collaboration (Udalski et al. 1994b, 2000; Woźniak et al. 2001), the MACHO collaboration (Alcock et al. 1997, 2000a) and the MOA collaboration (Sumi et al. 2003) yielded optical depths significantly higher than these estimates, suggesting that a fundamental revision of Galactic models might be necessary (Binney et al. 2000). However, the interpretation of the first results was difficult because of two effects. First, stars in the direction of the Galactic Bulge are not necessarily located in the Bulge itself so foreground and background contamination must be taken into account. Second, and more importantly, photometry of the numerous faint stars in the crowded Galactic Bulge fields is complicated by "blending" where the reconstructed stellar flux receives contributions from more than one star. This makes the effective number of monitored stars greater than the number of cataloged stars and generates a complicated relationship between real and reconstructed amplifications.

These problems can be largely avoided by considering only microlensing of clump giant stars. Such stars are identified by their well-defined position in the color-magnitude diagram. This position ensures that they are most likely in the Galactic Bulge. Their large flux also makes blending problems relatively unimportant.

The first published optical depth using clump giants was based on only 13 events observed by the MACHO group (Alcock et al. 1997). The optical depth, $3.9_{-1.2}^{+1.8} \times 10^{-6}$ at $\langle l, b\rangle=$ $\left(2.55^{\circ},-3.64^{\circ}\right)$, was still considerably higher than expectations. Since then, measurements using clump giants have tended to give results in better agreement with models. Based on 16 events, the EROS-2 collaboration (Afonso et al. 2003b) gave an optical depth of $0.94 \pm 0.29 \times 10^{-6}$ at $\langle l, b\rangle=\left(2.5^{\circ},-4.0^{\circ}\right)$. The MACHO group (Popowski et al. 2005) recently presented their results using 62 events with clump giant sources. Their optical depth is $\tau=2.17_{-0.38}^{+0.47} \times 10^{-6}$ at $\langle l, b\rangle=\left(1.5^{\circ},-2.68^{\circ}\right)$, in good agreement with calculations and with their earlier preliminary results (Popowski et al. 2000). The OGLE-II group (Sumi et al. 2006) recently used 33 events to obtain $\tau=2.55_{-0.46}^{+0.57} \times 10^{-6}$ at $\langle l, b\rangle=\left(1.16^{\circ},-2.75^{\circ}\right)$, again in agreement with models.

In this paper we present a measurement of the optical depth for microlensing toward the Galactic Bulge based on the totality of the EROS-2 clump-giant data. More details on this analysis can be found in Hamadache (2004). The 120 events used comprise the largest sample of clump-giant events studied to date. Section 2 describes the data collection and processing required to produce the light curves. Section 3 describes the definition of the clump-giant sample to be used to measure the optical depth. Section 4 lists the criteria used to isolate a sample of microlensing candidates. Section 5 describes the calculation of the detection efficiency, the optical depth and the timescale distribution. Section 6 discusses the possibility that stellar blending may affect the results. Finally, Sect. 7 compares our results with those of other groups and with the predictions of Galactic models.

\section{Data}

The data were acquired by EROS- 2 with the $1 \mathrm{~m}$ MARLY telescope at La Silla, Chile. The imaging was done simultaneously by two cameras, using a dichroic beam-splitter (Bauer et al. 1997). Each camera was composed of a mosaic of eight $2048 \times 2048$ pixel LORAL CCDs, with a pixel size of $0 . ' 6$ yielding a field of $0.7^{\circ}(\alpha) \times 1.4^{\circ}(\delta)$. Each camera observed through one of two non-standard, broadband filters, $R_{\text {eros }}$ and $B_{\text {eros }}$. The filter $R_{\text {eros }}$ covers the range $620-920 \mathrm{~nm}$ and has a mean wavelength near that of Cousins $I$, while $B_{\text {eros }}$ covers the range 420-720 $\mathrm{nm}$ and has a mean wavelength between Johnson/Cousins $V$ and $R$. The photometric calibration was obtained directly for $20 \%$ of our fields by matching our star catalogues with those of the OGLE-II collaboration (Udalski et al. 2002). To a precision of $\sim 0.1 \mathrm{mag}$, we find

$R_{\text {eros }}=I_{\text {ogle }}, B_{\text {eros }}=V_{\text {ogle }}-0.4(V-I)_{\text {ogle }}$.

The calibration is sufficiently uniform that it can be extended with confidence to the remaining fields. We note, however, that none of our conclusions depend on the calibration precision.

The observations reported here concern 66 fields, monitored between July 1996 and October 2002. Figure 1 shows the location of the 66 fields in Galactic coordinates, $(l, b)$. Twenty-two of the fields are in the north $(b>0)$ and 44 in the south. The corresponding data set contains $6 \times 10^{7}$ light curves, of which $5.6 \times 10^{6}$ are Bulge clump giants that we use in the present analysis.

The fields are observable at La Silla from mid-February to mid-October. An average of one point every four nights was taken for each field during the observing season though the sampling frequency occasionally reached more than one point per night at mid-season when the Galactic Bulge is observable throughout the night.

The image photometry was performed with software specifically designed for crowded fields, PEIDA (Photométrie et Étude d'Images Destinées à l'Astrophysique) (Ansari 1996). The first step consisted of creating reference images for each of the 2112 subfields of size $\sim 0.035 \mathrm{deg}^{2}$, i.e. one quarter of a CCD. A reference image was constructed by adding 15 high-quality 


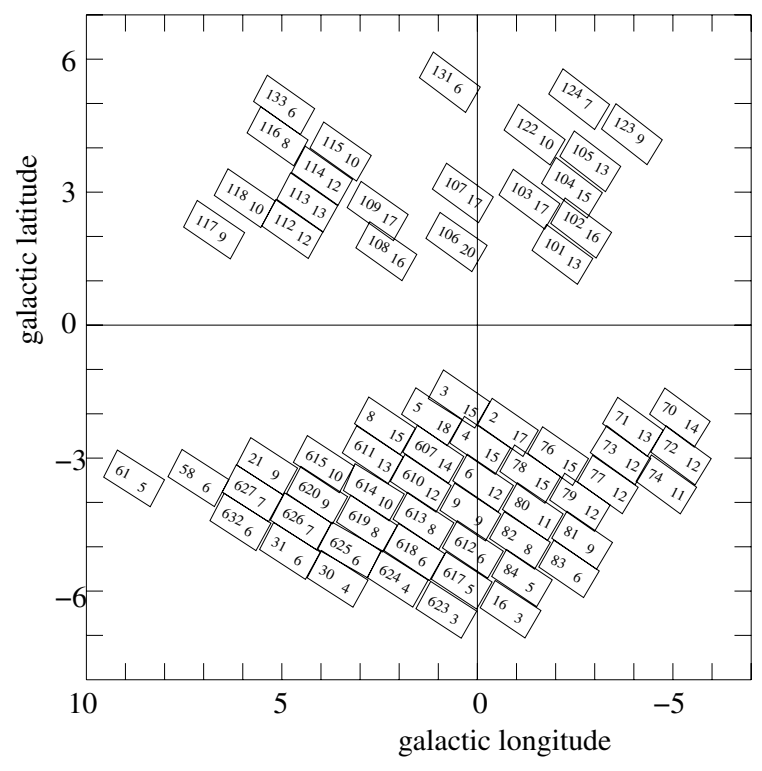

Fig. 1. Map of the EROS-2 Bulge fields in Galactic coordinates. A total of 66 fields were monitored. The first number in each field is the field number and the second is the number of clump giants in the field divided by $10^{4}$. The field 121 with $4 \times 10^{4}$ clump giants is outside the plot at $(l, b)=(11.5,2.5)$.

images of the subfield. Star catalogs for each subfield were established using the reference image. Photometry on individual images was then performed by imposing the stellar positions found on the reference image.

A first estimate of the uncertainty of individual flux measurements is that due to the photometric fit taking into account only photon counting statistics. This clearly underestimates the uncertainty since it ignores other contributions important in crowded field photometry, e.g. imperfect knowledge of the point-spreadfunction (PSF). The factor by which the uncertainties must be increased is determined by comparing individual flux measurements with the flux measurements of the same star on the reference image. The fit errors are increased by a magnitude and image dependent (but star independent) factor, chosen so that for each image the distribution of the difference between flux and reference flux is consistent with that expected from the renormalized flux uncertainties. By construction, this procedure yields, for most stars, a $\chi^{2}$ per degree of freedom near unity for a fit to a time-independent flux.

The uncertainties on flux measurements are reflected in the dispersions of light curves about the baseline flux (estimated from the mode of the flux). Figure 2 shows, for each subfield, the median dispersion for clump giants in the two colors. The photometric precision is typically $2 \%$ but degrades considerably for $B_{\text {eros }}$ in highly absorbed fields. We will be concerned only with microlensing apparent amplification factors $>1.6$ so the precision in most fields is sufficiently good to yield high quality light curves in both colors. The $20 \%$ of the microlensing candidates with the lowest quality light curves were also photometered with our differential photometry package (Le Guillou 2004) so as to confirm their microlensing origin.

Examples of light curves containing microlensing events are shown in Figs. 21-23.
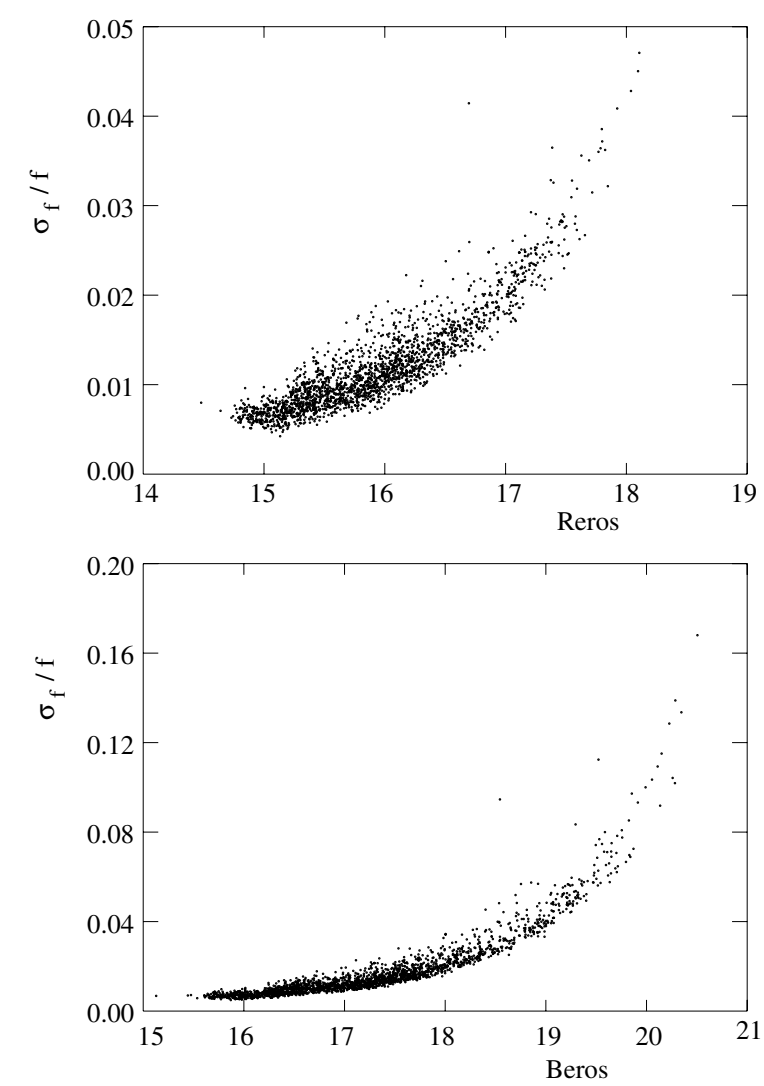

Fig. 2. The dispersion, $\sigma / f_{\text {base }}$, of clump-giant light curves about the base flux as a function of $R_{\text {eros }}$ and of $B_{\text {eros }}$. Each point represents the median dispersion of clump giants for one subfield consisting of a CCDquarter.

\section{Clump giant selection}

The sample of clump-giants to be studied was selected using the color-magnitude diagrams for each quarter-CCD subfield. Figure 3 shows a diagram of a typical subfield. The center of the clump was determined by modeling the density of stars in colormagnitude space as a power law representing a smooth background plus a Gaussian representing the clump. The Gaussian had the form

$$
\begin{aligned}
\text { density } & \propto \exp \left[-\frac{\left((B-R)_{\text {eros }}-\alpha\right)^{2}}{2 \sigma_{\alpha}^{2}}\right] \\
& \times \exp \left[-\frac{\left(R_{\text {eros }}+1.9(B-R)_{\text {eros }}-\beta\right)^{2}}{2 \sigma_{\beta}^{2}}\right]
\end{aligned}
$$

where $\alpha\left(\sigma_{\alpha}\right)$ and $\beta\left(\sigma_{\beta}\right)$ are the desired clump centers (widths) in the space defined by $(B-R)_{\text {eros }}$ and the reddening-free magnitude $R_{\text {eros }}-1.9(B-R)_{\text {eros. }}$. The dereddened magnitude is used so as to be relatively insensitive to variable reddening across the subfields.

Figure 4 a shows the fitted $R_{\text {eros }}$ of the clump center as a function of the fitted $(B-R)_{\text {eros }}$ of the clump center. The distribution follows the expected reddening law indicating that variations of clump position are due to differential reddening. The 46 subfields that are outliers in this plot are excluded from further consideration leaving 2063 subfields. All 2063 color-magnitude diagrams were visually inspected to ensure that reasonable values for the clump position were found. We note that, in spite of the large variation of clump position, the clump is visually distinct 


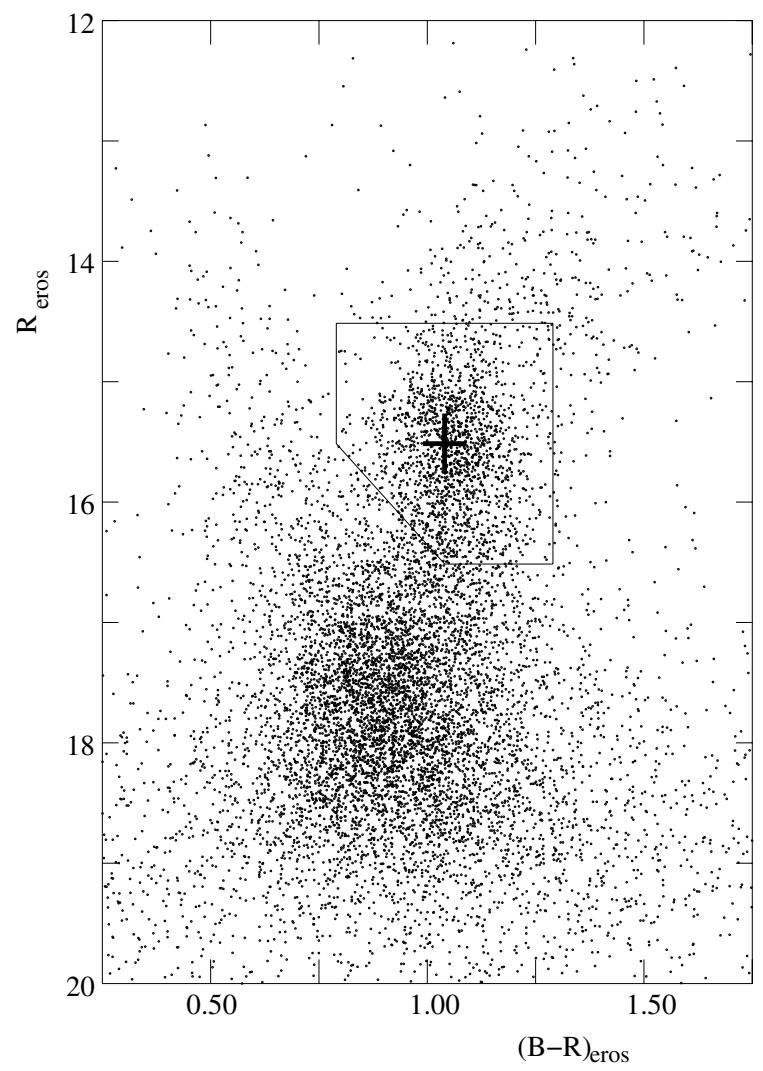

Fig. 3. A typical color-magnitude diagram of a subfield (field 610, CCD 4 quarter 2). The cross shows the fitted center of the clump and the thin lines show the cuts defining the clump giants used in this analysis.

in each of the 2063 diagrams. The variations of clump position lead to varying photometric precisions (Fig. 2) but this is taken into account in the microlensing detection efficiency (Sect. 5).

Figure $4 \mathrm{~b}$ shows the dereddened clump magnitude as a function of Galactic longitude, $\ell$. The longitude dependence is believed to be caused by the bar structure of the Bulge where stars with $l>0$ are brighter than stars with $l<0$ because they are nearer (Stanek et al. 1994). The 0.38 mag difference over $12^{\circ}$ in longitude corresponds to a bar orientation of $49^{\circ} \pm 8^{\circ}$ with respect to the line of sight to the Galactic Center. This angle is in agreement with the original OGLE-I results (Stanek et al. 1994) and with the recent infrared star counts from GLIMPSE (Benjamin et al. 2005)

Figure 5a shows the clump color as a function of Galactic latitude. The northern fields are more absorbed and reddened than the southern fields.

The cuts used to define our sample of clump giants, illustrated in Fig. 3, are

$\left|R-R_{\text {clump }}\right|<1$,

$\left|(B-R)-(B-R)_{\text {clump }}\right|<0.25$,

$R-R_{\text {clump }}>-4 .\left[(B-R)-(B-R)_{\text {clump }}\right]-1$,

where $R_{\text {clump }}$ and ( $\left.B-R\right)_{\text {clump }}$ define the clump center. The number of clump giants as a function of Galactic latitude is shown in Fig. 5b. As expected, the number falls rapidly with increasing distance from the Galactic equator. In spite of the difference in absorption between northern and southern fields, there is no obvious asymmetry between counts of northern and southern clump giants, as expected.
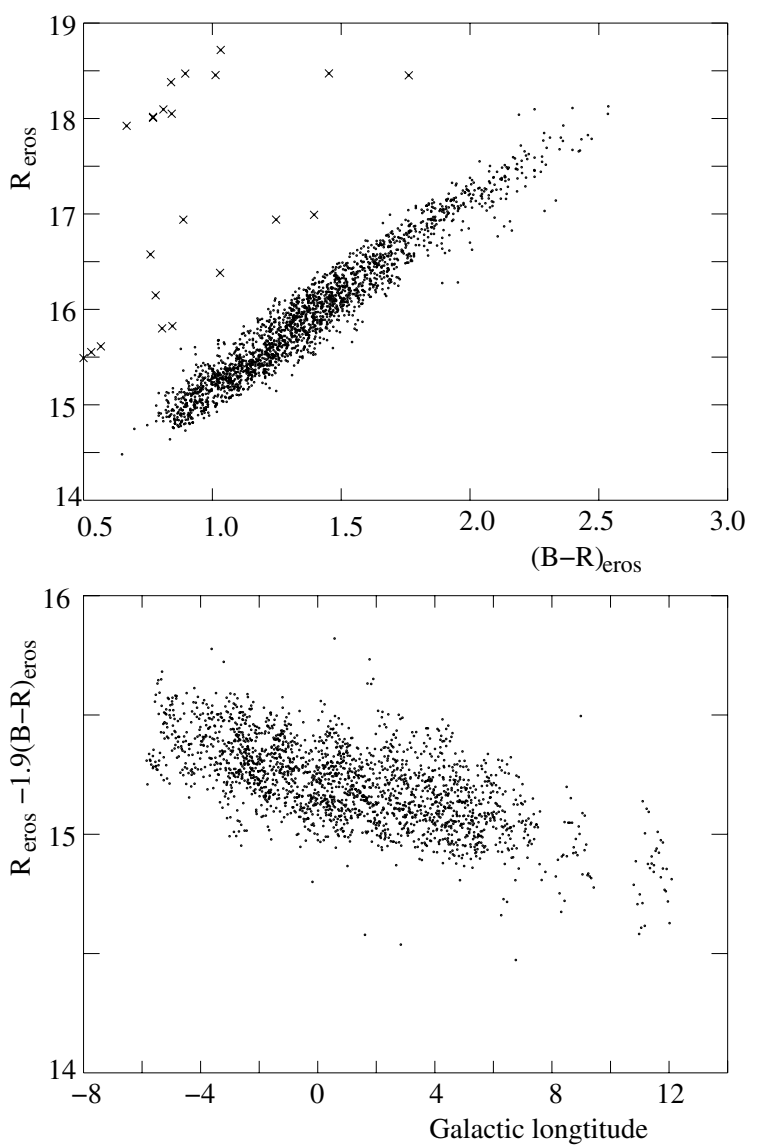

Fig. 4. The top panel shows $R_{\text {eros }}$ of the clump center vs. $B_{\text {eros }}-R_{\text {eros }}$ of the clump for the 2109 subfields (CCD quarters). (Three quarters were not reconstructed.) The distribution follows the expected reddening law. Quarters marked with $\mathrm{a} \times$ were eliminated from further consideration. The bottom panel shows the dereddened magnitude as a function of Galactic longitude. The increasing flux with longitude is believed to be due to the bar structure since stars with $l<0$ are farther than stars with $l>0$.

\section{Event selection}

After the production of the light curves, microlensing candidates among the $5.6 \times 10^{6}$ clump giants were found by the procedures described in this section. After elimination of images with instrumental problems or poor seeing, the first step was to calculate the mode of the red and blue fluxes that was used as a first estimate of the baseline fluxes.

The light curves were then subjected to a "filter" that selects light curves having one or more groups of points with fluxes sufficiently above the baseline flux, $f_{\text {base }}$. Such groups were initiated with any point $i$ having a flux $f_{i}$ greater than $f_{\text {base }}+2.5 \sigma_{i}$ where $\sigma_{i}$ is the estimated uncertainty of $f_{i}$. The groups ended with the first subsequent sub-group of three points all with $f_{i}<f_{\text {base }}+2 \sigma_{i}$. The relative significance of the groups on a light curve was defined by

$L P_{N}=N \log 2-\sum_{i=1}^{N} \log \left[\operatorname{erfc}\left(\frac{f_{i}-f_{\text {base }}}{\sqrt{2} \sigma_{i}}\right)\right]$,

where $N$ is the number of points in the group. For each red or blue light curve, groups having at least 5 points with $f_{i}>$ $f_{\text {base }}+2.5 \sigma_{i}$ were then ordered by decreasing significance. A light curve was then retained for further analysis if one of the 

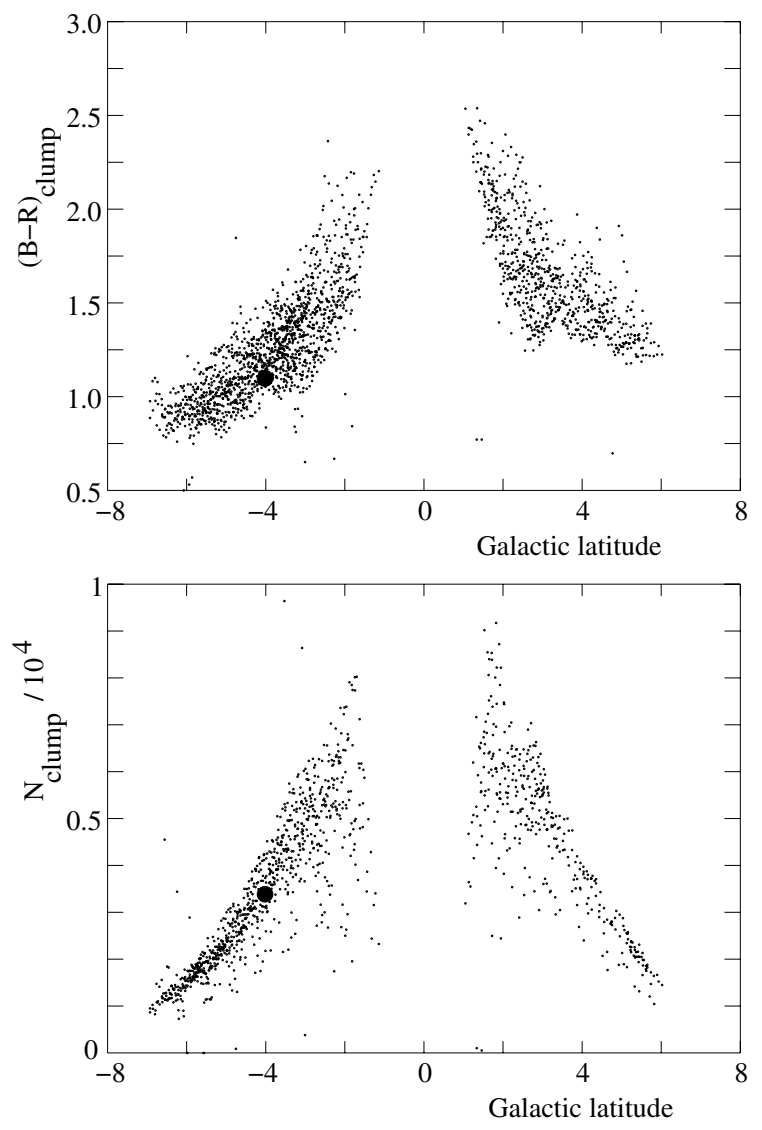

Fig. 5. The mean clump color (top panel) and the number of clump giants per quarter CCD (bottom panel) as a function of Galactic latitude for the longitude range $-3^{\circ}<\ell<3^{\circ}$. The number of giants is symmetric about $b=0$ while northern fields are more reddened than southern fields. The large dot marks the position of the subfield containing the Baade Window.

two most significant red groups had a temporal overlap of at least $20 \%$ with one of the two most significant blue groups.

Roughly $3 \%$ of the light curves pass this filter. The filter's effective threshold is sufficiently low that, for clump stars, the filter passes essentially all microlensing events with apparent amplifications greater than $30 \%$ occurring during well-sampled parts of the observing period. Significant losses of efficiency occur due to bad weather, equipment failure and limited visibility of the Galactic Bulge at the beginning and end of each season.

Light curves passing the filter were then fit in each color with the microlensing light curve corresponding to uniform motion of an unblended point source and single lens. The total flux as a function of time is

$f(t)=f_{\text {base }} \frac{u^{2}+2}{u \sqrt{u^{2}+4}}$

where the impact parameter $u(t)$ is

$u^{2}(t)=u_{0}^{2}+\frac{\left(t-t_{0}\right)^{2}}{t_{\mathrm{E}}^{2}}$.

For each color, there are 4 fitted parameters: the baseline flux $f_{\text {base }}$, the time of maximum apparent amplification $t_{0}$, the impact parameter at maximum amplification normalized to the Einstein ring radius $u_{0}=u\left(t_{0}\right)$, and finally, the microlensing event duration, i.e. the Einstein ring radius crossing time
$t_{\mathrm{E}}=r_{\mathrm{E}} / v_{\mathrm{t}}$ for a transverse relative velocity $v_{\mathrm{t}}$. The last two quantities depend on the Einstein radius, $r_{\mathrm{E}}^{2}=4 G M D_{\mathrm{l}}\left(D_{\mathrm{s}}-D_{\mathrm{l}}\right) / c^{2} D_{\mathrm{s}}$, where $M$ is the mass of the lens, and $D_{1}$ and $D_{\mathrm{s}}$ are the distances to the lens and source, respectively.

While all transient flux variations can be fitted more or less successfully with the above expression, ideal microlensing events are characterized by achromaticity, i.e. the same $u_{0}, t_{\mathrm{E}}$ and $t_{0}$ in all pass-bands. Because of the rarity of the phenomenon, one generally also expects the events to be not repeated for a given star. A final important characteristic is that the detection efficiency corrected distribution of $u_{0}$ is flat. These characteristics guide the final selection of microlensing candidates. This selection is based on seven criteria, $\mathrm{C} 1-\mathrm{C} 7$, applied to the parameters of the microlensing fit performed on light curves passing the filter. The cuts we use lead to a detection efficiency that is relatively independent of $u_{0}$ so the flat distribution of $u_{0}$ is only moderately distorted. On the other hand, the cuts necessarily result in an efficiency that depends on $t_{\mathrm{E}}$ since very short events have a significant chance of falling between observations. The cuts are also sufficiently loose to maintain a good efficiency for finding non-conventional microlensing events, e.g. those due to binary lenses.

The first five criteria, $\mathrm{C} 1-\mathrm{C} 5$, are applied independently to the parameters of the fits for both bands and events are required to pass the criteria in both. $\mathrm{C} 1$ requires that the time of maximum apparent amplification be within the observing season so that the microlensing fit will yield reliable lensing parameters:

C1: $\quad t_{0} \in$ observing season.

The beginning and end of the seven observing seasons were defined separately for each of the 66 fields as the period between the first and last successful images for that field. For each field, only seasons with a mean sampling interval of less than 8.5 days are used. Sub-fields covered by CCD 2 were ignored for the first 4 seasons when the red CCD-2 was mostly out of order.

The second cut eliminates very long candidate events:

$$
\text { C2: } \quad t_{\mathrm{E}}<400 \mathrm{~d} \text {. }
$$

A visual scan of events eliminated by this cut indicates that they are generally low amplitude events $\left(u_{0}>0.8\right)$. No high amplitude microlensing events $\left(u_{0}<0.5\right)$ were seen. Many of the $t_{\mathrm{E}}>400 \mathrm{~d}$ events appear to be simple baseline shifts, with most occurring when the telescope optics were realigned at $t=\mathrm{JD}-2450000=983$. Most stars with such shifts are near very bright stars and it is probable that the shifts are due to slight changes in the wings of the PSF.

The third cut eliminates events due to known equipment problems. Most importantly, we eliminate long events occurring before $t=\mathrm{JD}-2450000=900$ by requiring

$$
\text { C3: } \quad t_{e}<100 \mathrm{~d} \text { if } t_{0}<900 .
$$

A visual scan of the $\sim 5000$ events eliminated with this cut indicates that almost all are due to the aforementioned baseline shifts at $t=983$ which yielded events with $t_{0} \sim 800$. We also eliminated 48 events on CCDs 6 and 7 between days 2323 and 2343 that were due to an electronics problem.

The next cut requires sufficient sampling during the event

C4: $\quad \geq 4$ points with $\left|t-t_{0}\right|<t_{\mathrm{E}}$,

and $\quad \geq 8$ points with $\left|t-t_{0}\right|<2 t_{\mathrm{E}}$.

This cut has the largest influence on the $t_{\mathrm{E}}$ dependence of the efficiency. 


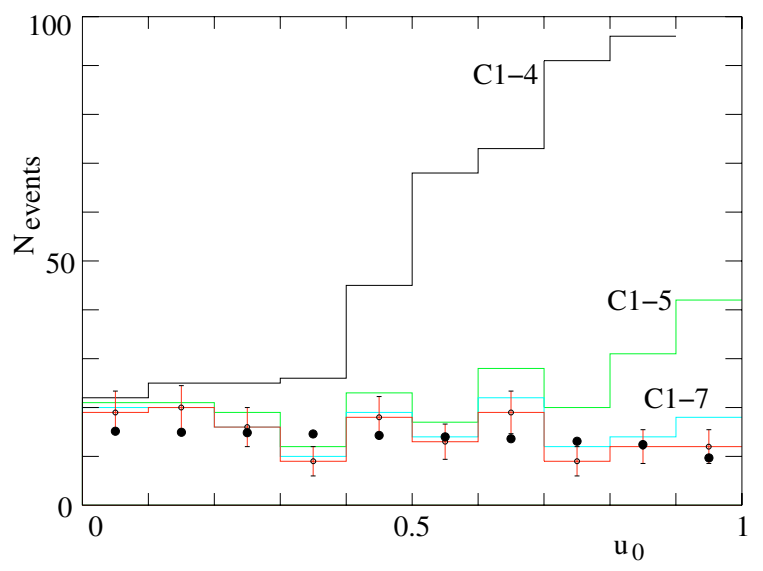

Fig. 6. The evolution of the $u_{0}$ distribution with the applied candidate selection criteria. The first histogram is for the 577 events with $u_{0}<1.0$ satisfying criteria $\mathrm{C} 1, \mathrm{C} 2, \mathrm{C} 3$ and $\mathrm{C} 4$. The second is for the 234 events that satisfy the five criteria $\mathrm{C} 1-\mathrm{C} 5$ and the third for the 165 events satisfying the seven criteria $\mathrm{C} 1-\mathrm{C} 7$. In the final histogram, 18 events have been eliminated whose light curves show additional variations outside the main variation or are clearly baseline shifts. The dots give the Monte-Carlo distribution for microlensing events satisfying all criteria.

Cut 5 is a very loose cut on the $\chi^{2}$ of the microlensing fit calculated using only the points far from the time of maximum amplification, $\left|t-t_{0}\right|>2 t_{\mathrm{E}}$ :

C5: $\quad \chi_{\text {base }}^{2} / N_{\text {d.o.f. }}<10$.

This cut eliminates most periodic and chaotic variable stars but retains single excursion events, even those that fit badly the simple light curve (8), e.g. events due to binary lenses.

Cuts 6 and 7 require reasonable agreement between the blue and red light curves:

$$
\begin{array}{ll}
\text { C6: } & \frac{\left.t_{0} \text { (blue }\right)-t_{0}(\text { red })}{\left\langle t_{\mathrm{E}}\right\rangle}<0.4, \\
\text { C7: } & \frac{t_{\mathrm{E}}(\text { blue })-t_{\mathrm{E}}(\text { red })}{\left\langle t_{\mathrm{E}}\right\rangle}<0.3 .
\end{array}
$$

For the most part, these two cuts eliminate short time-scale excursions due to photometric problems on single exposures.

To understand the effect of each cut it is interesting to follow the evolution of the $u_{0}$ distribution as successive cuts are applied. The first distribution in Fig. 6 is for the 577 events with $u_{0}<1.0$ that pass cuts $\mathrm{C} 1, \mathrm{C} 2, \mathrm{C} 3$ and $\mathrm{C} 4$. The distribution is dominated by events with $u_{0}>0.5$ corresponding to low amplitude variations. This is indicative of contamination by low amplitude variable stars. However, already with just these cuts, the distribution is flat for $u_{0}<0.4$ indicating that there is little background for high apparent amplification microlensing events.

The second distribution is for the 234 events that also satisfy C5. This eliminates most of the variable stars, and the $u_{0}$ distribution is now quite flat for $u_{0}<0.8$. Application of cuts C6 and C7 eliminate a few additional events, mostly near $u_{0} \sim 1$, leaving 165 events with a distribution that is flat for $u_{0}<1.0$.

The 165 light curves were then visually examined. Most events fit well the simple microlensing light curve though a significant fraction show interesting effects. A summary of the scan conclusions is given in Table 1 for the 129 events with $u_{0}<0.75$ where there is generally little ambiguity about the nature of the events. Non-simple microlensing events include four
Table 1. The tentative classifications of the 129 events satisfying cuts $\mathrm{C} 1-\mathrm{C} 7$ and $u_{0}<0.75$. The symbols in parentheses (B, P, X, C,?) show how these classifications are designated in Fig. 8 and Table 3. The last column gives the relative weight of each classification if they were used in the calculation of the optical depth using the $t_{\mathrm{E}}$ from the simplelens fit. (In reality, only 120 events are used, eliminating the 3 single excursion (?) events and 6 chaotic/periodic variables or baseline shifts.)

\begin{tabular}{lll}
\hline \hline Event type & Number & $\sum\left(t_{\mathrm{E}} / \epsilon\right) /$ total \\
\hline simple & 104 & 0.77 \\
strong blends $(\mathrm{B})$ & 5 & 0.02 \\
strong parallax $(\mathrm{P})$ & 4 & 0.11 \\
binary non caustic $(\mathrm{X})$ & 2 & 0.02 \\
caustic (C) & 5 & 0.06 \\
single excursion (?) & 3 & 0.01 \\
chaotic/periodic variable & & \\
or baseline shift & 6 & - \\
\hline
\end{tabular}

strong parallax events, i.e. those with light curves strongly affected by the circular motion of the Earth $(\mathrm{P})$. There are five binary lens events with visible caustic crossings (C). Five events show strong source blending (B) and are discussed in more detail in Sect. 6. Two events (X) show achromatic deviations from the simple lens curve that might be due either to non-uniform motion of the source in a binary system (i.e. a so-called xallarap event) or to a binary lens without caustic crossing. Six events show chaotic or periodic variations outside the primary variation and are therefore clearly not microlensing events. Finally three events marked (?) show single excursions that fit badly the simple lens curve but could conceivably be due to binary lenses.

The events with $u_{0}>0.75$ are visually not very striking, and a non-negligible number were eliminated by scanning. We therefore choose to eliminate these events so that for the remainder of this paper, we consider only the 120 events selected by $\mathrm{C} 1-\mathrm{C} 7$ and classified as microlensing events with $u_{0}<0.75$, i.e. maximum amplifications $A_{\max }>1$.6. The characteristics of the 120 events are listed in Table 3. Figures 21-23 show the light curves of three candidate events, the first with the most significant improvement in $\chi^{2}$ over the constant-flux light curve, the second with the median improvement, and the third with the least significant improvement. All of the 120 light curves can be found on our web page, http: //eros.in2p3.fr

The $u_{0}$ distribution for the selected events is quite flat, as can be seen in Fig. 6. It agrees with the expected distribution from the Monte-Carlo simulation. The fact that the observed and expected distributions fall slightly near $u_{0}=1$ is due to the effect of the event filter that requires a sufficient number of points significantly above the baseline. Figure 7 shows the cumulative $u_{0}$ distribution for the 120 events and the efficiency-corrected curve for a flat $u_{0}$ distribution (with the observed distribution of $t_{\mathrm{E}}$ ). The Kolgomorov-Smirnov test gives a CL of $83 \%$. As discussed in Sect. 6 , the sample may have a $\sim 10 \%$ contamination at large $u_{0}$ by blended events due to the microlensing of faint background stars. Eliminating any randomly selected 10 events in the expected range $0.5<u_{0}<0.75$ yields a CL of $34 \%$, still quite acceptable.

The distribution of $\chi^{2}$ per degree of freedom calculated for points in the range $\left|t-t_{0}\right|<2 t_{\mathrm{E}}$ is shown in Fig. 8. The simple events have a reasonable distribution with a mean value of 1.24 , a quite satisfactory value considering the difficulty of estimating photometric uncertainties. The other categories of events have, naturally, larger values of reduced $\chi^{2}$, especially caustic events (C). 


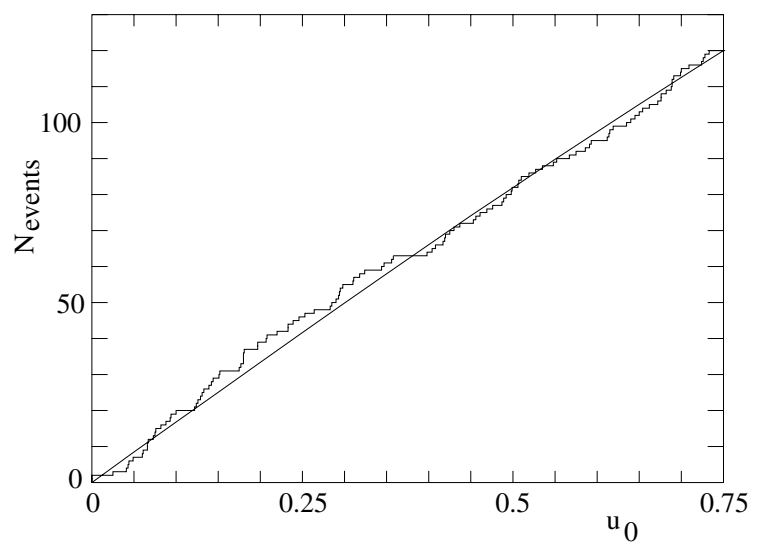

Fig. 7. The cumulative $u_{0}$ distribution for the 120 selected events. The smooth curve shows the efficiency-corrected flat $u_{0}$ distribution.

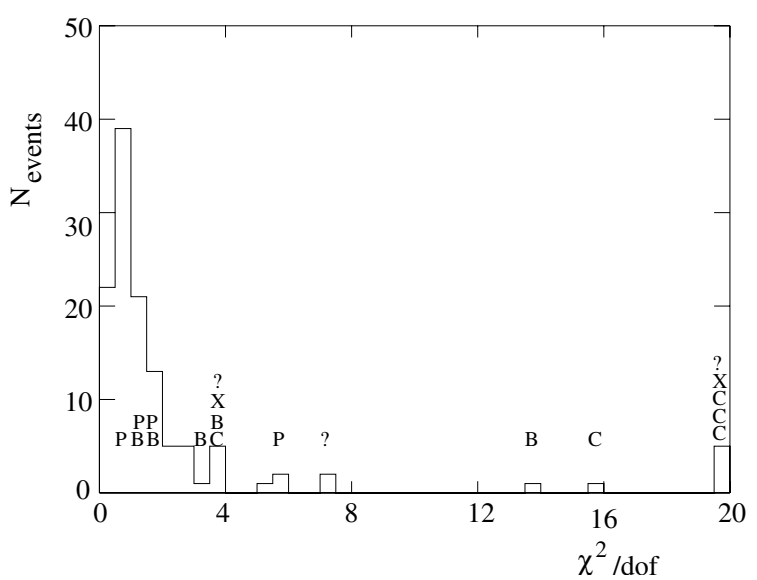

Fig. 8. The distribution of the reduced $\chi^{2}$ calculated using points within the range $\left.\mid t-t_{0}\right) \mid<2 t_{\mathrm{E}}$. The 120 accepted events plus the 3 (?) events appear in the plot. The letters show the positions of the 19 non-simple events as described in Table 1.

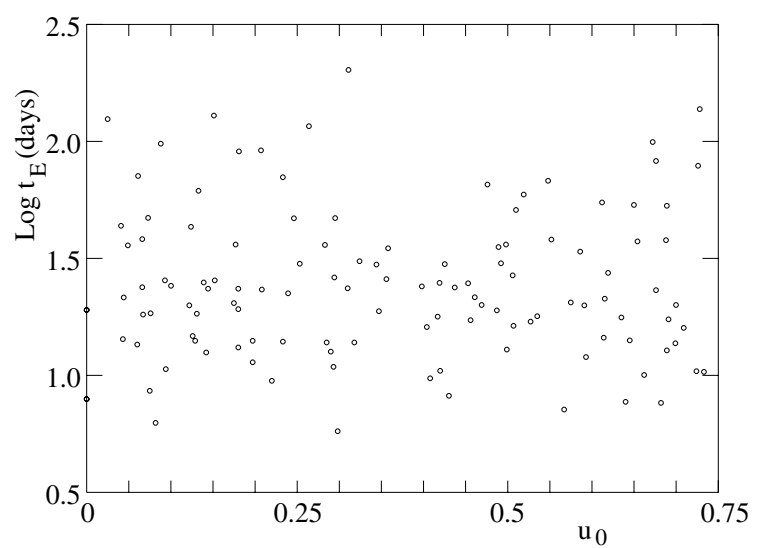

Fig. 9. The distribution of $\left(u_{0}, t_{E}\right)$ for the 120 events passing all selection criteria with $u_{0}<0.75$.

Figure 9 shows the scatter plot of $\log \left(t_{\mathrm{E}}\right)$ vs. $u_{0}$. The $u_{0}$ distribution is, as expected, not strongly dependent on $t_{\mathrm{E}}$. Figure 10 shows the distribution of event magnitude and color relative to the center of the clump. The events show a distribution that is very similar to that of the ensemble of clump stars.
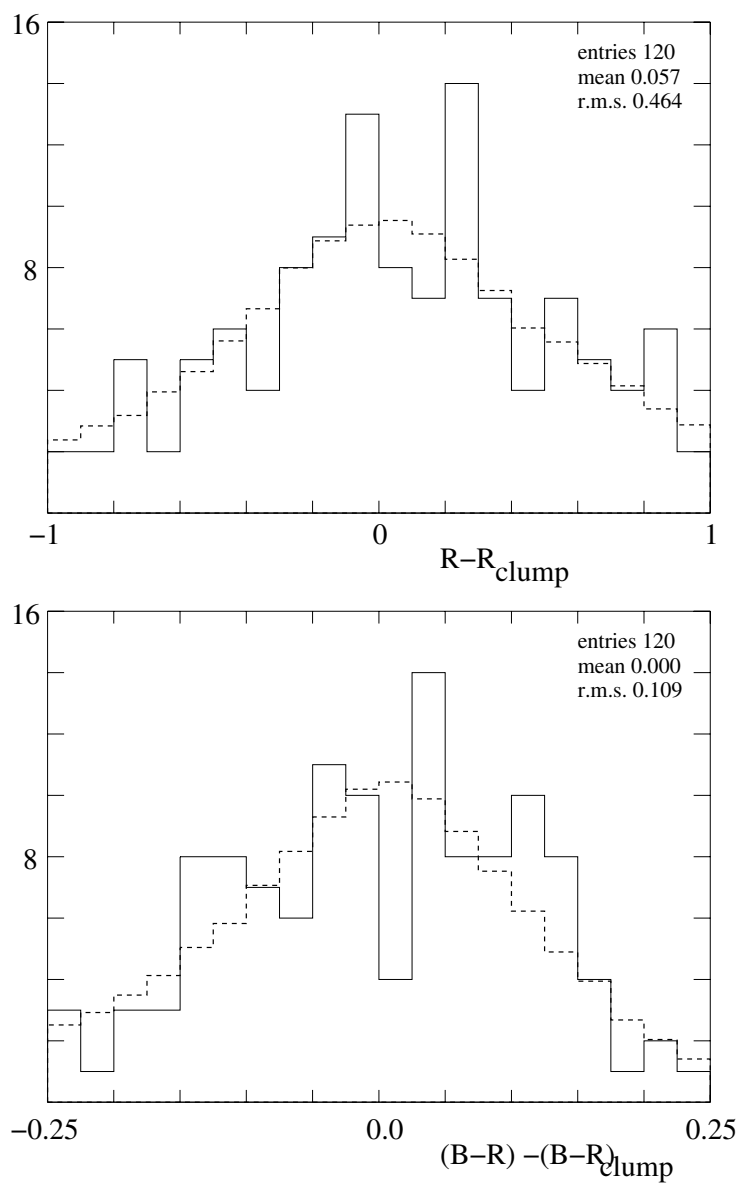

Fig. 10. The top panel shows the distribution of $R_{\text {eros }}$ relative to the center of the clump, $R_{\text {clump }}$. The bottom panel shows the distribution of $(B-R)_{\text {eros }}$ relative to the center of the clump, $(B-R)_{\text {clump. In both cases, }}$ the dashed histogram shows the distribution of random clump stars.

The events in the sample are generally well described by the light curve (8), as indicated by the reduced $\chi^{2}$ distribution (Fig. 8). Further indication that the events are indeed microlensing events comes from their achromaticity and time symmetry. Figure 11 shows that the events have equal $u_{0}$ and $t_{\mathrm{E}}$ in the blue and red as expected for microlensing events with negligible blending. Figure 12 shows that the events have equal rise and fall times. This test for time symmetry was performed by fitting the light curves with the form (8) with $t_{\mathrm{E}}$ in Eq. (9) modified by

$t_{\mathrm{E}} \rightarrow \Delta t=t_{\mathrm{E}}\left[1+\alpha \arctan \left(\frac{t-t_{0}}{t_{\mathrm{E}}}\right)\right]$.

The parameter $\alpha$ describes the asymmetry of the light curve with $\alpha=0$ corresponding to a symmetric (microlensing) curve and $\alpha>0(\alpha<0)$ corresponding to rise times less than (greater than) the fall time. As an example, supernova light curves generally give $\alpha>0.3$. The observed distribution (Fig. 12) is symmetrically peaked at $\alpha=0$ as expected for microlensing events.

\section{Optical depth and $t_{\mathrm{E}}$ distribution}

To determine the optical depth and the distribution of $t_{\mathrm{E}}$, we must first evaluate the detection efficiency as a function of time scale $t_{\mathrm{E}}$ by using Monte-Carlo simulated light curves. We superimpose artificial microlensing events, with randomly generated 

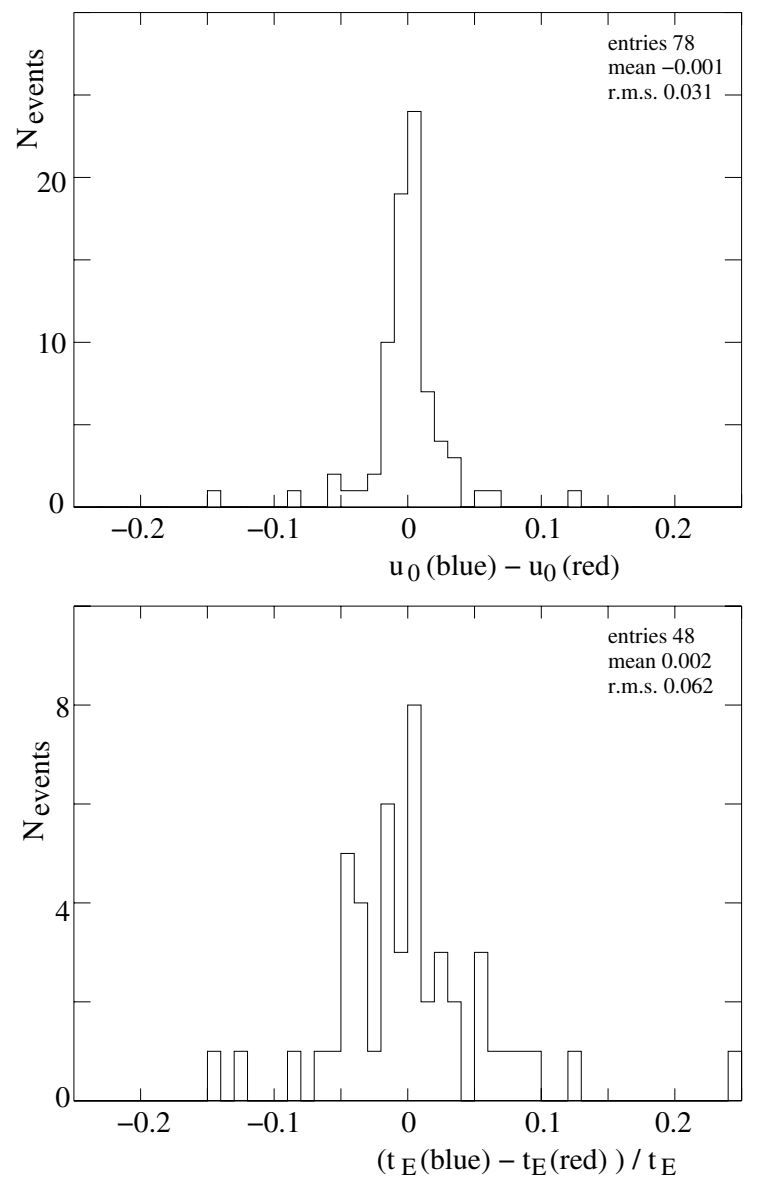

Fig. 11. Tests for achromaticity of the events. The top panel shows the difference between red and blue minimum impact parameters, $u_{0}$, for the 78 events with at least three points in the range $t_{0}-t_{\mathrm{E}} / 2<t<$ $t_{0}+t_{\mathrm{E}} / 2$ for both red and blue curves, ensuring a precise measurement of the maximum amplification in both colors. The bottom panel shows the difference between red and blue $t_{\mathrm{E}}$ for the 48 events with at least three points in the range $t_{0}-3 t_{\mathrm{E}} / 2<t<t_{0}-t_{\mathrm{E}} / 2$ and three points in the range $t_{0}+t_{\mathrm{E}} / 2<t<t_{0}+3 t_{\mathrm{E}} / 2$ for both red and blue curves, ensuring a precise measurement of $t_{\mathrm{E}}$ in both colors.

parameters (impact parameter, date of maximum amplification and time scale), on a random sample of real light curves. These light curves are then subjected to the same microlensing search as the original light curves and we determine the fraction that are recovered by our detection algorithm. More precisely, the detection efficiency as a function of $t_{\mathrm{E}}$ is given by the ratio between two numbers:

- The number of events passing all selection criteria and with the reconstructed $t_{\mathrm{E}}$ within the considered $t_{\mathrm{E}}$ bin and with the reconstructed $u_{0}<0.75$.

- The number of generated microlensing events with generated $t_{\mathrm{E}}$ within the considered $t_{\mathrm{E}}$ bin, with generated $t_{0}$ within the observing period for the field in question and with the generated $u_{0}<0.75$.

Figure 13 shows the efficiency as a function of $t_{\mathrm{E}}$ for a subsample of individual fields. The efficiency is field-independent at the $10 \%$ level. For $t_{\mathrm{E}}>100 \mathrm{~d}$ criterion C3 causes a slight discontinuity in the efficiency for fields that were well observed during the first two seasons.

The microlensing optical depth is defined as the probability that a given star, at a given time $t$, is magnified by at least 1.34 ,

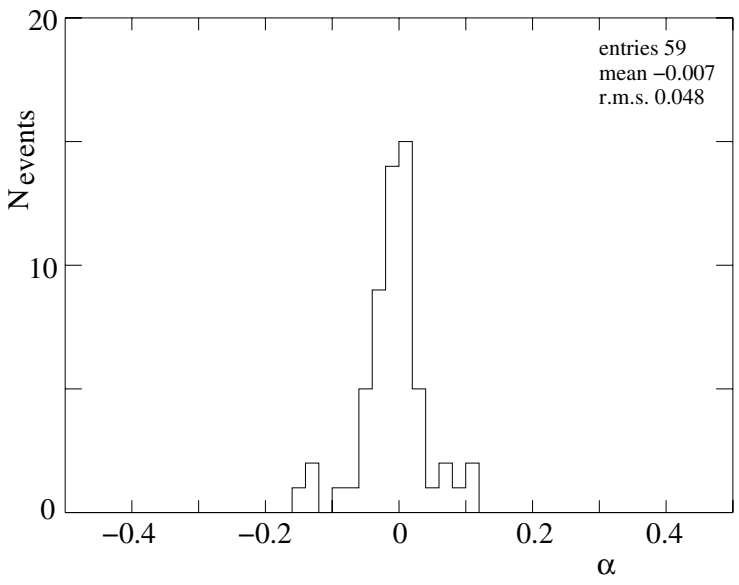

Fig. 12. Test for time symmetry of the events. The histogram is of the parameter $\alpha$ defined by (10) for events with $t_{0}$ at least 50 days away from both the beginning and the end of the observing season, ensuring a good measurement of $\alpha$.

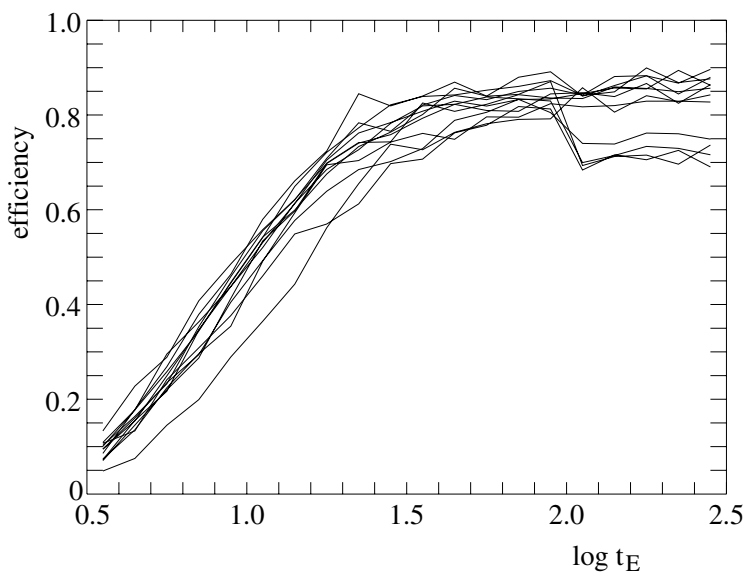

Fig. 13. Detection efficiency for individual fields as a function of the event time scale $t_{\mathrm{E}}$ (in days) for $u_{0}(\max )=0.75$. For the sake of clarity, only $1 / 4$ of the 66 fields are shown. The discontinuity at $t_{\mathrm{E}}=100 \mathrm{~d}$ is due to criterion $\mathrm{C} 3$ for fields that were observed during the first two seasons.

i.e. with an impact parameter $u(t)<1$. The optical depth is then given by

$\tau=\frac{\pi}{2 u_{0}(\max )} \frac{\sum_{i=1}^{N_{\mathrm{ev}}} t_{\mathrm{E}, \mathrm{i}} / \epsilon\left(t_{\mathrm{E}, \mathrm{i}}\right)}{\sum_{j=1}^{N_{\star}} T_{j}}$,

where $N_{\star}$ is the number of monitored stars, $T_{j}$ is the observation period for star $j, t_{\mathrm{E}, \mathrm{i}}$ is the measured Einstein crossing time of the $i$ th candidate and $\epsilon\left(t_{\mathrm{E}, \mathrm{i}}\right)$ is the detection efficiency. For each event, the efficiency is taken from the corresponding subfield consisting of one CCD (Fig. 13).

The denominator of $(11)$ is $6.62 \times 10^{9} \mathrm{star} \cdot$ days. Using the 120 events in Table 3, we find a calculated optical depth averaged over all fields which is

$\tau=1.68 \pm 0.23 \times 10^{-6}, \quad\langle|b|\rangle=3.43^{\circ}$.

The uncertainty is mostly statistical, estimated following the prescription of Han \& Gould (1995)

$\sigma(\tau)=\tau \frac{\sqrt{\left\langle t_{\mathrm{E}}^{2} / \epsilon^{2}\right\rangle}}{\left\langle t_{\mathrm{E}} / \epsilon\right\rangle} \frac{1}{\sqrt{N_{\mathrm{ev}}}}=0.21 \times 10^{-6}$. 


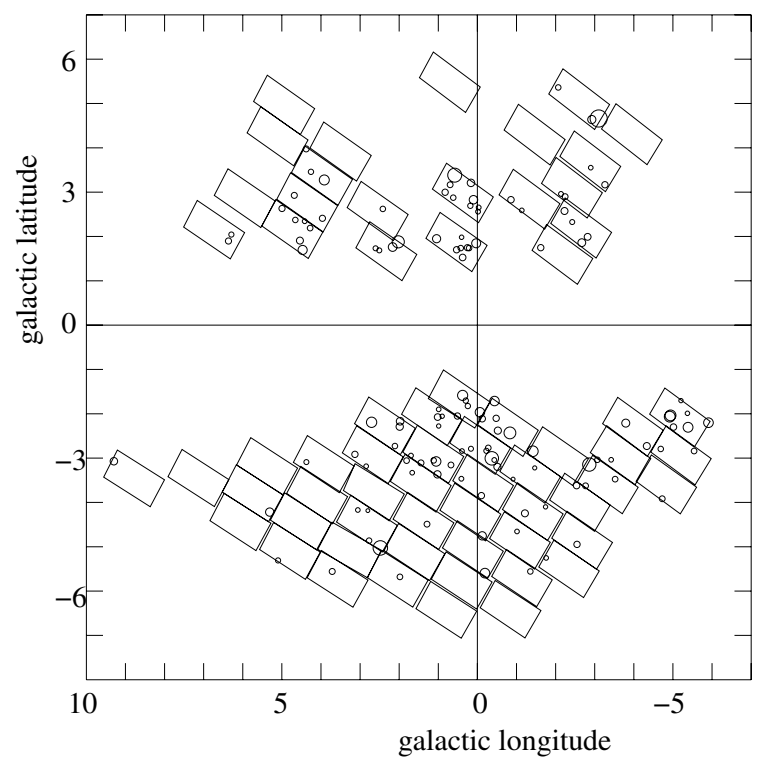

Fig. 14. The location of the 120 candidates used for the calculation of the optical depth. The area of each circle is proportional to the contribution of each candidate to the optical depth.

To the statistical error we have added in quadrature a $5 \%$ systematic uncertainty due to blending effects, as discussed in Sect. 6. The location and contribution of each of the 120 candidates to the measured optical depth is shown in Fig. 14.

Because of the rapid variation of the optical depth across our fields, the above mean optical depth is of limited interest. Table 2 and Fig. 15 show the depth for various slices in Galactic latitude and longitude. The latitude gradient is clearly seen. Fitting the five points shown in Fig. 15a, one finds

$\tau / 10^{-6}=(1.62 \pm 0.23) \exp \left[-a\left(|b|-3^{\circ}\right)\right]$,

with

$a=(0.43 \pm 0.16) \mathrm{deg}^{-1}$.

At $b=-2.7^{\circ}$ this corresponds to an optical depth gradient of $(0.78 \pm 0.27) \times 10^{-6} \mathrm{deg}^{-1}$, in agreement with the less precise values of MACHO (Popowski et al. 2005) $(1.06 \pm 0.71) \times 10^{-6} \mathrm{deg}^{-1}$ and of OGLE-II (Sumi et al. 2006) $(0.78 \pm 0.84) \times 10^{-6} \mathrm{deg}^{-1}$. We see no significant difference between the northern and southern fields, as expected.

The dependence on Galactic longitude is expected to be much weaker. Additionally, nonuniform placement of the observing fields makes it easy to mask a longitudinal dependence by the stronger latitude dependence. Figure $15 \mathrm{~b}$ shows the measured optical depth as a function of longitude for EROS-2 fields in the latitude range $1.4^{\circ}<|b|<3.0^{\circ}$. Also shown are predictions for our fields of various models. No significant longitudinal dependence is seen.

Figure 16 shows the $t_{\mathrm{E}}$ distribution of the 120 events, both raw and efficiency corrected. The mean $t_{\mathrm{E}}$ (Table 2) is

$\left\langle t_{\mathrm{E}}\right\rangle=(28.3 \pm 2.8) \mathrm{d}$.

We see no statistically significant latitude or longitude dependence of $\left\langle t_{\mathrm{E}}\right\rangle$ (Table 2). The north-south difference,

$\left\langle t_{\mathrm{E}}\right\rangle_{b>0}-\left\langle t_{\mathrm{E}}\right\rangle_{b<0}=(8.2 \pm 5.5) \mathrm{d}$,

is not significant. Figure 17 shows the cumulative $t_{\mathrm{E}}$ distribution of the 120 events showing their contribution to the optical depth;
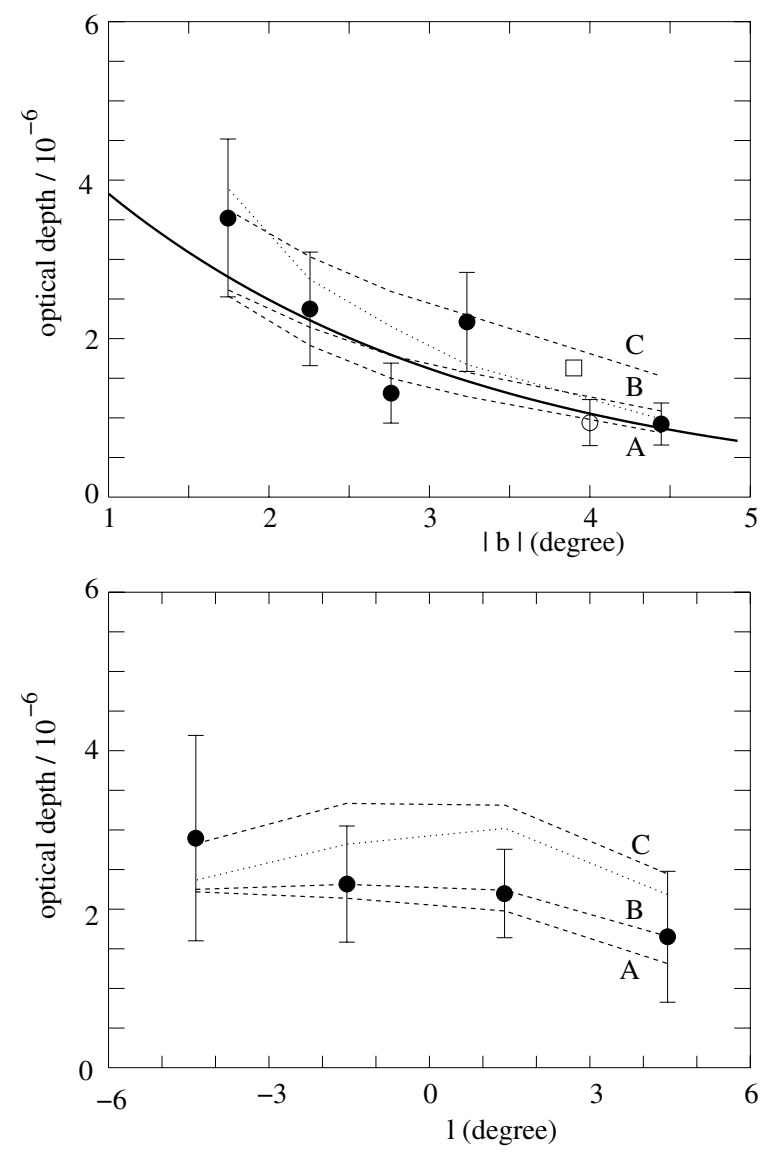

Fig. 15. The top panel shows the EROS-2 measured optical depth as a function of Galactic latitude. The bottom panel shows the optical depth as a function of Galactic longitude for the latitude range $1.4^{\circ}<|b|<$ $3.0^{\circ}$. The filled circles are from this work while the open circle in the upper panel is from the first EROS-2 analysis (Afonso et al. 2003b). In the upper panel, the solid line shows the fit (14). In both panels, the dotted lines show the prediction of the model of Bissantz \& Gerhard (2002) and the dashed lines, A, B and C, show the predictions of three models used by Evans \& Belokurov (2002) as described in Sect. 7. The open square in the upper panel is the prediction for the Baade Window by Han \& Gould (2003).

$10 \%$ of the optical depth comes from the 24 events with $t_{\mathrm{E}}<$ $13.6 \mathrm{~d}$ and $10 \%$ from the 4 events with $t_{\mathrm{E}}>120 \mathrm{~d}$.

The $15 \%$ error in the optical depth (Eq. (14)) and $10 \%$ error in $\left\langle t_{\mathrm{E}}\right\rangle$ (Eq. (16)) are mostly statistical, reflecting the number of events and the distribution of $t_{\mathrm{E}}$. We believe that it is unlikely that any systematic errors are this large.

The most obvious systematic error would come from possible contamination of the event sample with events not due to microlensing. This is a serious problem for microlensing searches in the Magellanic Clouds. We note however that the Magellanic microlensing rate is at least a factor 10 lower than in the Galactic Bulge. Furthermore, the identified Magellanic background events should not be a problem here since they concern stars that are not in the clump region (blue bumpers, Be stars) or background supernovae that have light curves sufficiently different from microlensing light curves to be easily identified for the high signal-to-noise ratio events considered here. At any rate, there is no indication that our sample of 120 events is contaminated with events not due to microlensing. Among the indications for lack of significant contamination are

- the $\chi^{2}$ distribution (Fig. 8) indicates that the function (8) is a good description of the light curves; 
Table 2. The measured optical depth for various slices in Galactic latitude $b$ and Galactic longitude $\ell$.

\begin{tabular}{lrrrllll}
\hline \hline Range & $\langle|b|\rangle$ & $N_{\text {stars }}$ & $N_{\mathrm{ev}}$ & $\left\langle t_{\mathrm{E}}\right\rangle$ & $\sigma\left(t_{\mathrm{E}}\right)$ & $\left\langle t_{\mathrm{E}}\right\rangle_{\text {cor }}$ & $\tau / 10^{-6}$ \\
\hline $1.40<|b|<7.00$ & 3.34 & 5569216 & 120 & 32.95 & 31.07 & $28.31 \pm 2.84$ & $1.68 \pm 0.22$ \\
& & & & & & & \\
$1.40<|b|<2.00$ & 1.75 & 630884 & 25 & 29.17 & 21.85 & $24.88 \pm 4.37$ & $3.52 \pm 1.00$ \\
$2.00<|b|<2.50$ & 2.26 & 829123 & 22 & 39.29 & 28.20 & $30.91 \pm 6.01$ & $2.38 \pm 0.72$ \\
$2.50<|b|<3.00$ & 2.76 & 976707 & 24 & 22.87 & 15.49 & $20.89 \pm 3.16$ & $1.31 \pm 0.38$ \\
$3.00<|b|<3.50$ & 3.23 & 931147 & 25 & 37.17 & 37.31 & $33.26 \pm 7.46$ & $2.21 \pm 0.62$ \\
$3.50<|b|<7.00$ & 4.45 & 2194599 & 24 & 36.75 & 41.51 & $32.60 \pm 8.47$ & $0.92 \pm 0.27$ \\
& & & & & & & \\
$-3.50<b<-1.40$ & 2.69 & 1861880 & 54 & 35.68 & 30.22 & $31.33 \pm 4.11$ & $2.42 \pm 0.47$ \\
$1.40<b<3.50$ & 2.50 & 1512735 & 42 & 27.27 & 23.42 & $23.08 \pm 3.61$ & $1.94 \pm 0.42$ \\
& & & & & & & \\
$-6.00<\ell<-3.00$ & 2.40 & 361293 & 10 & 46.02 & 28.13 & $39.43 \pm 8.89$ & $2.90 \pm 1.30$ \\
$-3.00<\ell<0.00$ & 2.42 & 660722 & 20 & 29.79 & 23.56 & $25.65 \pm 5.27$ & $2.32 \pm 0.73$ \\
$0.00<\ell<3.00$ & 2.22 & 981742 & 31 & 27.38 & 21.75 & $23.77 \pm 3.91$ & $2.20 \pm 0.56$ \\
$3.00<\ell<6.00$ & 2.53 & 316650 & 8 & 25.93 & 11.89 & $23.88 \pm 4.20$ & $1.65 \pm 0.83$ \\
\hline
\end{tabular}

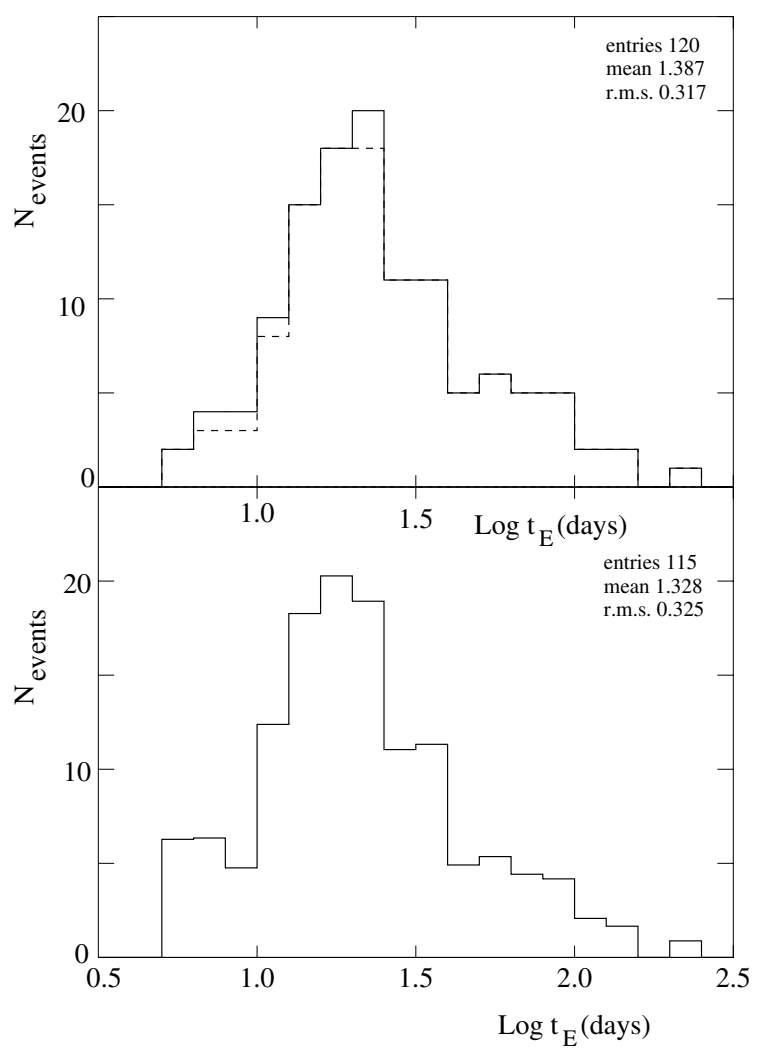

Fig. 16. The distribution of Einstein radius crossing times, $t_{\mathrm{E}}$. The top panel shows the raw distribution for the 120 events with $u_{0}<0.75$ with the dashed line corresponding to the 115 events that show no strong blending. The bottom panel shows the distribution corrected for the $t_{\mathrm{E}}$ dependence of the detection efficiency.

- the $u_{0}$ distribution is nearly flat as expected (Figs. 6 and 7);

- the events show no sign of chromaticity (Fig. 11);

- the light curves are symmetric about the time of maximum amplification (Fig. 12);

- the optical depth has the expected dependence on the Galactic latitude (Fig. 15). A background of variable stars might be expected to yield a measured optical depth that is latitude-independent.

A second systematic error could come from the contamination of the source sample by stars not in the Galactic Bulge. The number

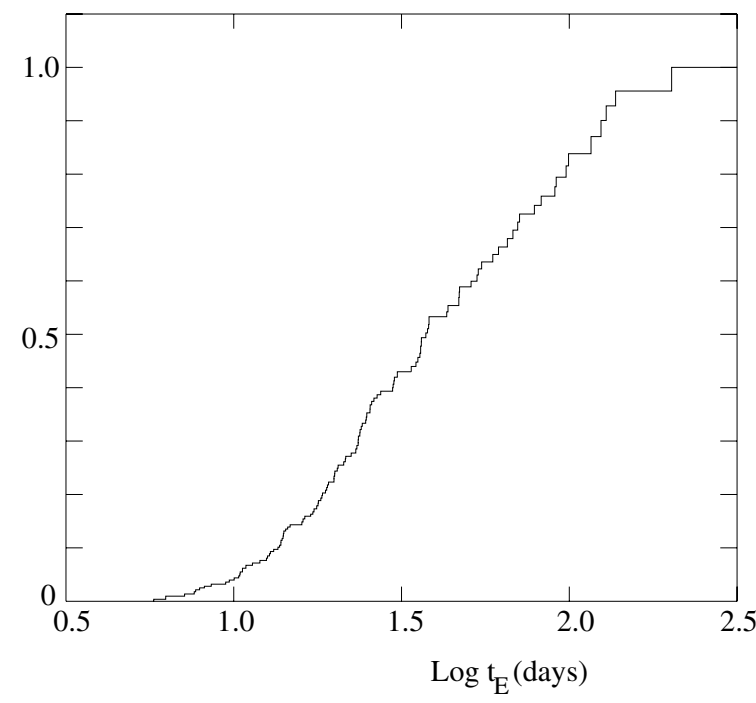

Fig. 17. The normalized cumulative distribution of the contribution of the 120 candidates to the observed optical depth as a function of $t_{\mathrm{E}} .10 \%$ of the optical depth comes from the 24 events with $t_{\mathrm{E}}<13.6 \mathrm{~d}$ and $10 \%$ from the 4 events with $t_{\mathrm{E}}>120 \mathrm{~d}$.

of source stars that are not in fact in the Galactic Bulge can be estimated from the general luminosity function and standard Galactic models. Generally, one finds that less than $1 \%$ of the stars in the clump box (Fig. 3) could be foreground main sequence stars. For negative latitudes, a contribution at the percent level can also be expected from bright giants in the Sagittarius dwarf galaxy where stars are 2.3 mag dimmer than low-latitude stars in the Galactic Bulge (Alard 1996).

A third effect comes from the preferential selection of source stars on the near side of the Bulge because the magnitude cut on source stars (Fig. 3) favors stars on the near side. Compared to a star at the Galactic center, stars on the near (far) side of the bulge are shifted upward (downward) in the color-magnitude diagram. Faint stars on the near side are moved into the accepted magnitude range while bright stars move out. Since there are more dim stars than bright stars, there is a net gain of stars on the near side. The opposite effect occurs on the far side where there is a net loss of stars. We used a Monte-Carlo calculation to estimate the mean position of our source stars and found that it is shifted towards us from the Galactic center by about $5 \%$ of the rms Bulge light 
of sight thickness. Since stars on the near side have a lower than average optical depth for bulge-bulge lensing, we can expect that the optical depth is underestimated by of order $5 \%$. This factor is not negligible compared to the statistical errors and any precise comparison with Galactic models will require that models properly weight source stars by their position in the Bulge.

A fourth effect could be the misclassification of non-standard microlensing events (e.g. caustic events) as variable stars. Our detection criteria are sufficiently liberal that several clear binary events were found, making up about $8 \%$ of the optical depth (Table 1). The ambiguous single excursion events would be only about $1 \%$ of the optical depth if they were included in the calculation. It therefore seems unlikely that there is a loss of events at the $10 \%$ level.

A fifth systematic error could come from the use of the $t_{\mathrm{E}}$ fitted with (8) for events due to non-standard lenses. The problem is minor for parallax events for which the modifications of formula (8) are relatively small and the use of the fitted $t_{\mathrm{E}}$ makes little difference. On the other hand, caustic events have light curves that resemble formula (8) only in the wings and the fitted $t_{\mathrm{E}}$ can be far from its true value, though for the caustic events in this study, the differences are only of order $10 \%$. At any rate, the number of events of this category is small, so we can expect that the modification of the calculated optical depth should be much less than $10 \%$. This was the conclusion of the detailed study of Glicenstein (2003).

The sixth systematic effect is that due to blending, the subject of the next section.

\section{Effect of blending on the measured optical depth}

Stellar blending complicates the interpretation of microlensing events in crowded fields. It is helpful to divide blending into two effects:

- Effect I: The photometry of bright (clump giant) stars is affected by the background of faint stars that are randomly placed with respect to the bright star. In the photometry, the sky background is assumed to be a smooth function so if the number of faint stars inside the seeing disk of the bright star is greater than the average number, the baseline flux of the bright star will be overestimated and the amplification due to microlensing underestimated. The opposite will occur if the number of faint stars is smaller than average. We can therefore expect that on average amplifications are correctly estimated. However, as emphasized in the previous section, the magnitude cut (Fig. 3) used to select source stars favors stars whose baseline fluxes are overestimated. We therefore expect a slight underestimation of the amplification.

- Effect II: The microlensing of a faint star within the seeing disk of a bright star will cause the reconstructed flux of the bright star to vary in time, yielding an apparent microlensing of the bright star.

By itself, effect II will clearly cause one to overestimate the optical depth if formula (11) is used since some of the events will not be due to the $N_{\text {star }}$ clump giants. On the other hand, we expect that effect I goes in the opposite direction since amplifications of clump giants are on average underestimated. Because of this, the reconstructed $t_{\mathrm{E}}$ found using formula (8) are systematically underestimated while the reconstructed $u_{0}$ are overestimated. In the formula (11) for the optical depth, the terms in the sum are therefore underestimated on average. Furthermore, fewer events are included in the sum because the overestimation of $u_{0}$ causes some events to migrate beyond $u_{0}(\max )$. Effect I therefore, by itself, causes the optical depth to be underestimated.

To estimate the importance of blending we have performed two types of tests with artificial stars placed on CCD images. The first type uses totally synthetic images in which all stars are artificial. This was used to quantify the sum of effects I and II. The second type uses real EROS-2 images in which artificial clump giants are added at random places on the images. This was used to quantify effect I.

The production and analysis of the totally synthetic images were described in the first EROS-2 Galactic Bulge article (Afonso et al. 2003b). Series of CCD images were produced by placing on them stars taken randomly from the luminosity distribution observed for the Baade window in HST deep images (Holtzman et al. 1998). The HST luminosity distribution extends to 9 mag fainter than clump giants and therefore allowed us to simulate the bright stars used in the EROS-2 analysis as well as the fluctuating background of faint stars. Microlensing events were assigned to a subsample of stars and the resulting flux change was taken into account when placing charge on the CCD. The sequence of CCD images were then reconstructed in the same way as normal EROS-2 images and events were searched for.

The results of this study indicated that reconstructed amplifications are indeed underestimated for clump giant stars leading to an underestimation of the optical depth (effect I) but that this is compensated to a precision of about $5 \%$ by events due to background stars (effect II). For the analysis of Afonso et al. (2003b), about $18 \%$ of the recovered optical depth was due to these faint stars compensating for the $15 \%$ underestimation for those events due to bright stars. For the analysis presented here, the effects are smaller because brighter stars are used and the amplification threshold is higher (1.6 instead of 1.34). We now find that only about $6 \%$ of the recovered optical depth is expected to be due to faint background stars.

Our second test used partially synthetic images on which we placed artificial clump giants on the real EROS-2 CCD images. As in the first test, microlensing events were simulated by giving the artificial giants time-dependent fluxes. These so-called spiked images were then photometered by the normal procedure and the events recovered and fitted with the microlensing light curve.

Figure 18 shows the comparison between recovered and simulated $t_{\mathrm{E}}$. This parameter is correctly estimated on average with $85 \%$ of events yielding a $t_{\mathrm{E}}$ within $20 \%$ of the generated $t_{\mathrm{E}}$. The $15 \%$ of events in the wings are due to stars whose baseline flux was strongly overestimated or underestimated because of blending. The nearly symmetric shape of the distribution in Fig. 18 means that a bias in the reconstruction of $t_{\mathrm{E}}$ results mostly from the fact that the magnitude cuts favor stars whose flux was overestimated. The distribution in Fig. 18 and the associated correlation with reconstructed baseline flux was thus combined with the observed magnitude distribution to make a Monte-Carlo estimate of the optical depth bias. The results confirm that effect II causes $\sim 5 \%$ underestimation of the optical depth.

Since these images only give information on effect II, we supplement it with a simple numerical calculation to estimate the contribution of faint stars in the seeing disk. A microlensed faint star superimposed on a bright star yields a light curve given by

$F(t)=F_{\mathrm{s}}\left[(1-f)+f \frac{u^{2}+2}{u \sqrt{u^{2}+4}}\right]$, 


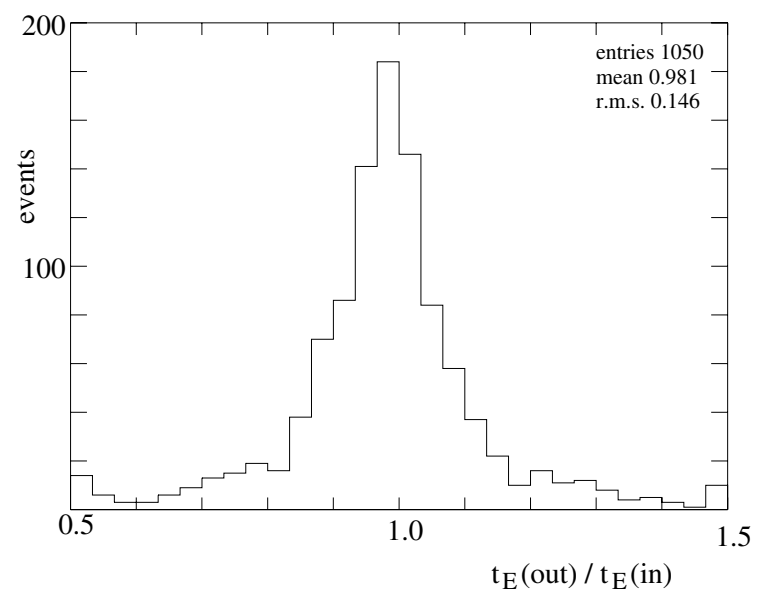

Fig. 18. The ratio of recovered and generated values of $t_{\mathrm{E}}$ for artificial clump giants placed on EROS-2 field 610.

where $f$ is the ratio between the faint star's baseline flux and the total reconstructed baseline flux. For $f \ll 1$, the reconstructed amplification is much less than the real amplification yielding reconstructed $u_{0}$ and $t_{\mathrm{E}}$ respectively much greater than and much less than the real $u_{0}$ and $t_{\mathrm{E}}$. In practice, this means that stars more than 4 mag fainter than clump giants yield no observable events because their reconstructed $t_{\mathrm{E}}$ are less than $5 \mathrm{~d}$. The HST luminosity function gives on average about 3 stars within 2 arcsec of a clump giant and within 4 mag of the clump magnitude. Integrating the HST function, we can expect that about $10 \%$ of clump giant events will be due to faint background stars. Since the events are much shorter than real clump giant events, they contribute only about $4 \%$ to the measured optical depth, not far the above estimates.

Because of the approximate compensation, we make no blending correction to the optical depth. Instead, because of the difficulty in estimating the effect precisely, we choose to include a $5 \%$ uncertainty in the optical depth, i.e. an uncertainty equal to the estimated size of each of the compensating effects. This uncertainty is, at any rate, less than the statistical uncertainty of the present measurements. Future high statistics measurements of the optical depth will certainly have to be much more careful about blending, especially if faint source stars are used.

Some strongly blended events can be found by fitting light curves to a microlensing curve (18). Since all events are blended to some extent, the number of detected blends is a sensitive function of the photometric precision. Five EROS-2 events showed a significant improvement for this curve over that with the simple curve (8). The events are characterized by excess amplification in their wings due to the fact that the real $t_{\mathrm{E}}$ is greater than the $t_{\mathrm{E}}$ fitted with Eq. (8).

The absence of strong blending in most events is seen in Fig. 19a showing $t_{\mathrm{E}}$ (blend) vs. $t_{\mathrm{E}}$ (no-blend). While the cloud of points is very wide because of the degeneracies in the fit, for the most part there is no systematic difference between the two $t_{\mathrm{E}}$, whereas $t_{\mathrm{E}}$ (blend) $>t_{\mathrm{E}}$ (no-blend) for truly blended events. This is confirmed in Fig. 19b, which shows the logarithm of the ratio of the two $t_{\mathrm{E}}$ 's both for the observed events and for the simulated clump giants. The observed distribution is very similar to the Monte-Carlo distribution.

The absence of strong blending for our events is not inconsistent with the results of the OGLE-II group who observed a large number of strongly blended events on clump giants.
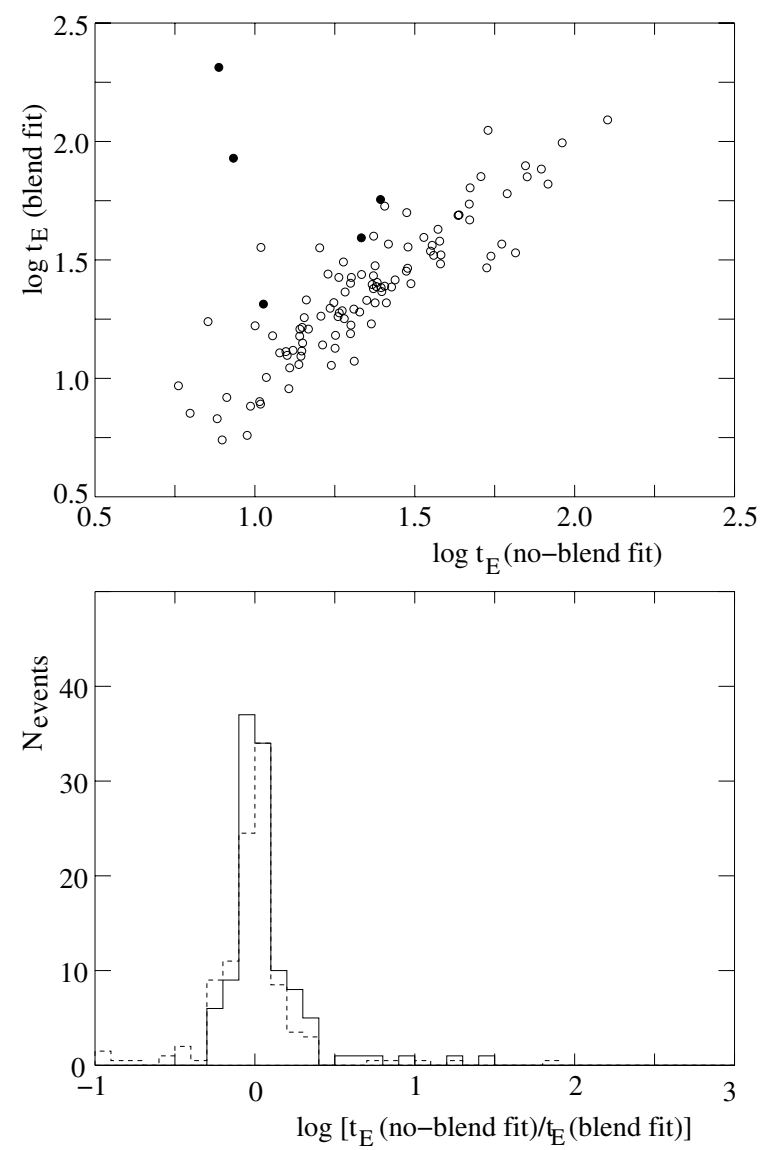

Fig. 19. The fitted $t_{\mathrm{E}}$ assuming blending (Eq. (18)), vs. the fitted value assuming no blending (Eq. (8)). The filled circles correspond to the five events showing strong blending. The bottom panel shows the logarithm of the ratio of the two $t_{\mathrm{E}}$ for simulated events (solid line) and observed events (dashed line).

Most of the OGLE-II blended events are very low amplification events, $\left(u_{0}\right.$ (no blend) $\left.>1\right)$ with only 4 of their 26 events with $u_{0}$ (no blend) $<0.75$ being characterized as blends. This proportion of blends is somewhat higher than our estimates, a fact that may be due to their superior photometric precision. At any rate, the number is sufficiently small to be of minor importance for optical depth determinations with $u_{0}<0.75$.

\section{Discussion and conclusions}

The EROS-2 optical depth measurement presented here is in remarkably good agreement with the other clump-giant measurements shown in Fig. 20, i.e. those of the MACHO group (Popowski et al. 2005) and OGLE-II group (Sumi et al. 2006). Only the original low statistics MACHO measurement (Alcock et al. 1997) is a bit too far from our fitted optical depth (14).

Figure 15 shows that our optical depth measurements are in reasonable agreement with some recent calculations based on models of the Galactic Bar and Disk. The dotted line in Fig. 15 is the prediction of the non-parametric model of Bissantz \& Gerhard (2002). The strong latitude dependence is correctly predicted as is the nearly flat predicted longitude dependence. The three dashed lines correspond to calculations of Evans \& Belokurov (2002) based on the three models of the inner Galaxy. Curve A is based on the model of Binney et al. (1997), curve B on the model of of Dwek et al. (1995), and curve $C$ on the model of of Freudenreich (1998). (For these three 


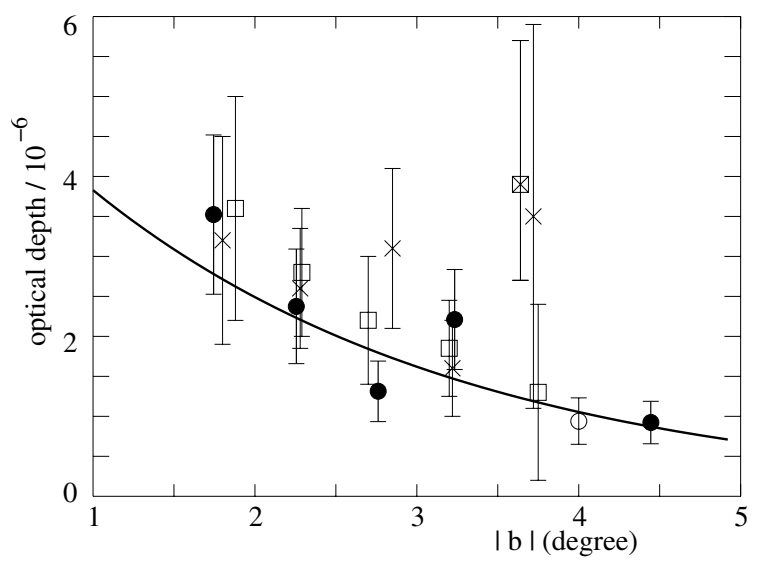

Fig. 20. Optical depth as a function of Galactic latitude. The filled circles are this work while the open circle is the first EROS-2 analysis (Afonso et al. 2003b). The solid line shows the fit (14). The measurements of the MACHO group are the open squares (Popowski et al. 2005) and the crossed square of the early analysis of Alcock et al. (1997). The crosses are from the OGLE-II group (Sumi et al. 2006).

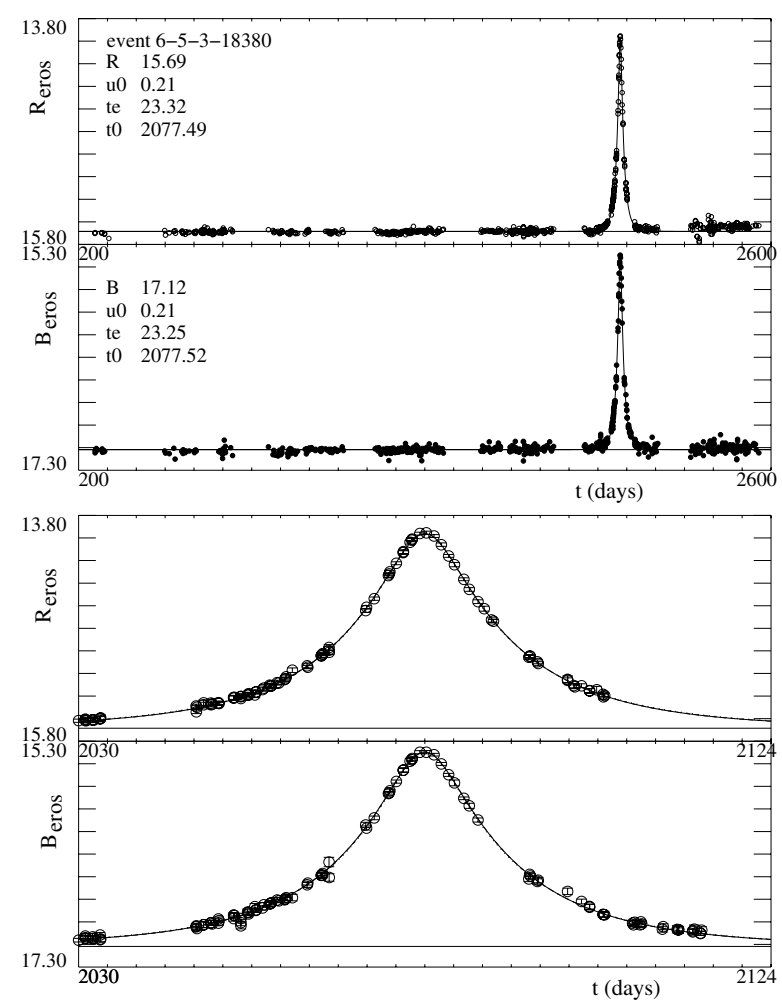

Fig. 21. The light curves of EROS-2 microlensing candidate 6-5-318380. Of the 120 candidates, this one has the largest value of $\chi^{2}$ (flat) $\chi^{2}$ (microlensing) $=221600$. The two top panels show $R_{\text {eros }}$ and $B_{\text {eros }}$ as a function of time (JD - 2450000). The two bottom panels show a zoom for the time interval $t_{0}-2 t_{\mathrm{E}}<t<t_{0}+2 t_{\mathrm{E}}$.

models, we show the predictions without corrections due to spiral structure; these would give at most a $20 \%$ increase to the calculated optical depth.) All three models have a common total mass of $1.5 \times 10^{10} M_{\odot}$ within $2.5 \mathrm{kpc}$ of the Galactic Center. The data in Fig. 15 indicate that the "swollen bar" model of Freudenreich (1998) is not favored by the data, unless the assumed mass is reduced by $\sim 30 \%$.

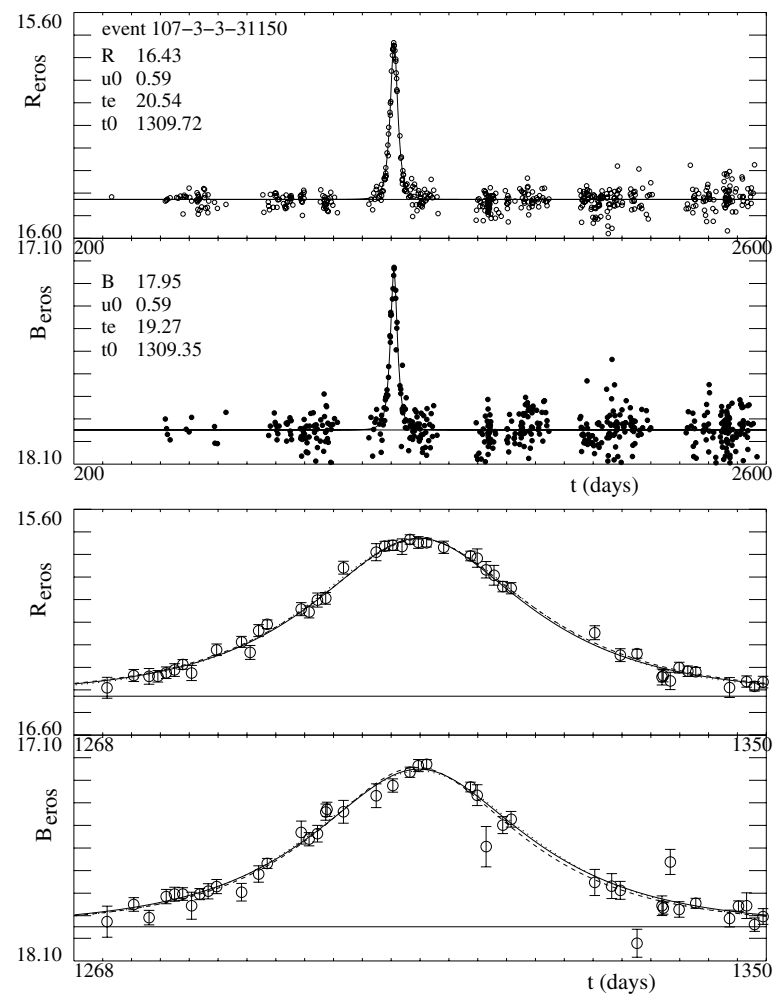

Fig. 22. The light curves of the EROS 2 microlensing candidate 1073-3-31150. Of the 120 candidates, this one has the median value of $\chi^{2}$ (flat) $-\chi^{2}$ (microlensing) $=6810$. The two top panels show $R_{\text {eros }}$ and $B_{\text {eros }}$ as a function of time (JD - 2450 000). The two bottom panels show a zoom for the time interval $t_{0}-2 t_{\mathrm{E}}<t<t_{0}+2 t_{\mathrm{E}}$. On the bottom two panels, the solid line is the blend-free simultaneous fit for both colors, the dashed line the blend-free single-color fit, and the dotted line the simultaneous fit with blending.

Finally, Fig. 15 shows the Baade window prediction of Han $\&$ Gould (2003). The calculated optical depth is within one standard deviation of the curve given by (14).

We make no attempt here to separate the Disk (2) and Bulge (3) contributions so our conclusion is simply that the model of Bissantz \& Gerhard (2002) or models A and B of Evans $\&$ Belokurov (2002) give a good estimate of the total optical depth (1). We note that these models place the bulk of the mass in normal stars and gas so the agreement with our measurements indicate that there is no need for additional mass in a non-lensing form. In models with cold dark matter, the dark component may make a significant contribution to the mass in the inner parts of the Milky Way. For example, in the models of Klypin et al. (2002), the cold dark matter contribution to the rotation curve at $1 \mathrm{kpc}$ from the Galactic Center is between $30 \%$ and $50 \%$. Such a contribution would, by itself, lower the optical depth by roughly the same factor. Our optical depth measurement, with its $15 \%$ uncertainty, therefore provides a useful constraint on such models.

Galactic models generally suppose a smooth distribution of lenses and therefore predict a smooth longitude and latitude dependence of the optical depth. The MACHO group (Popowski et al. 2005) observed 9 events near $\left(\ell=2.9^{\circ}, b=-2.9^{\circ}\right)$ (their field 104), yielding an optical depth about 2 standard deviations above the expectation based on their other fields. As seen in Fig. 14, we do not see an excess in this direction. Of their 9 events, 4 events occurred after the beginning of EROS-2. 


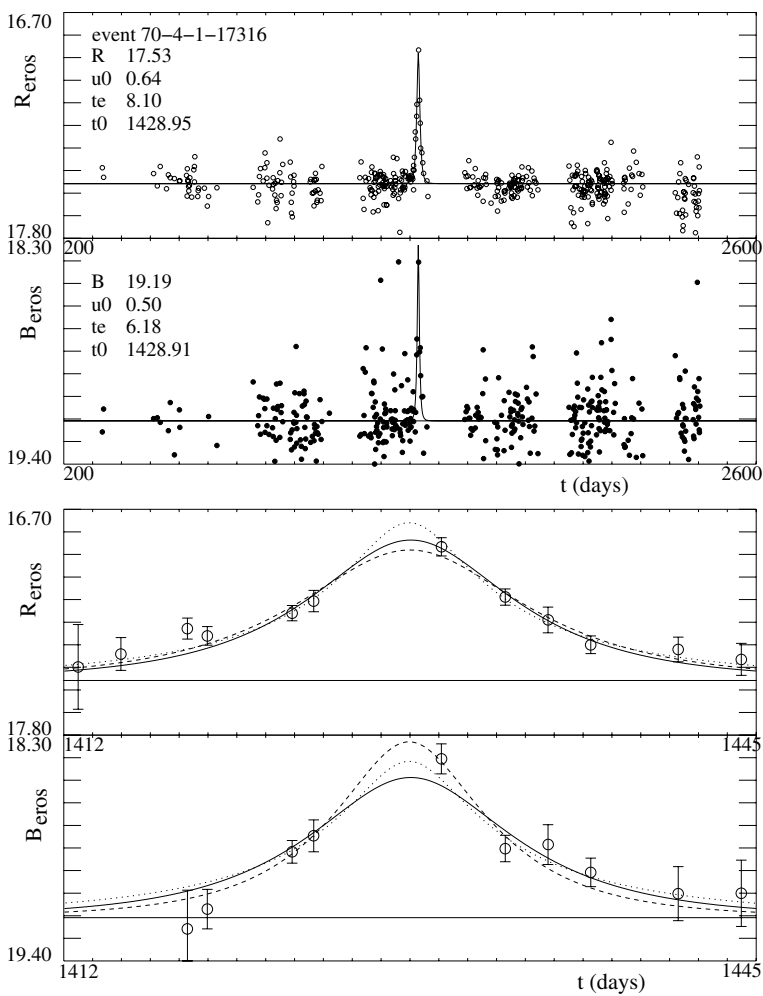

Fig. 23. The light curves of the EROS 2 microlensing candidate 704-1-14316. Of the 120 candidates, this one has the smallest value of $\chi^{2}$ (flat) $-\chi^{2}$ (microlensing) $=399$. The two top panels show $R_{\text {eros }}$ and $B_{\text {eros }}$ as a function of time (JD - 2450000). The two bottom panels show a zoom for the time interval $t_{0}-2 t_{\mathrm{E}}<t<t_{0}+2 t_{\mathrm{E}}$. On the bottom two panels, the solid line is the blend-free simultaneous fit for both colors, the dashed line the blend-free single-color fit, and the dotted line the simultaneous fit with blending.

Of these 4 events, two are not in an Eros field and two are in our field 611. Both of these events were found (in Table 3, MACHO events 104.20515.498 and 104.20640.8423). No other EROS events were found in this region in the three bulge seasons after the shutdown of MACHO. The fact that EROS-2 saw only two events for a solid angle and time period equivalent to that in which MACHO found 9 events suggests that the MACHO excess is a statistical fluctuation.

While our optical depth measurement is in good agreement with other measurements and with Galactic models, agreement on the $t_{\mathrm{E}}$ distribution is less satisfactory. Our value of $\left\langle t_{\mathrm{E}}\right\rangle$ is in excellent agreement with the value, $(28.1 \pm 4.3) \mathrm{d}$, found by OGLE-II (Sumi et al. 2006). The EROS-2 and OGLE-II values are, however, significantly higher than that calculated using the 62 MACHO events (Table 3 of Popowski et al. 2005): $\left\langle t_{\mathrm{E}}\right\rangle=(21.6 \pm 3) \mathrm{d}$. The discrepancy lies entirely in the large number of MACHO events with $t_{\mathrm{E}}<10 \mathrm{~d}$. The number of MACHO events with $\left(t_{\mathrm{E}}<5,5<t_{\mathrm{E}}<10, t_{\mathrm{E}}>10 \mathrm{~d}\right)$ is $(6,14,42)$ whereas the corresponding numbers for EROS-2 are $(0,10,110)$. Part of the differences in event numbers is due to the fact the the MACHO efficiency is relatively greater than the EROS-2 efficiency for $t_{\mathrm{E}}<10 \mathrm{~d}$. Using the $14 \mathrm{MACHO}$ events with $5<t_{\mathrm{E}}<10$ and the relative efficiencies of the two experiments, we estimate that EROS- 2 should see $24 \pm 7$ events in this range compared to the 10 that are seen. The very low EROS-2 efficiency for $t_{\mathrm{E}}<5 \mathrm{~d}$ makes it unsurprising that we found no events in this range.
Whatever the source of the discrepancy, these short events have relatively little effect on the measured optical depths. The events with $t_{\mathrm{E}}<10 \mathrm{~d}$ constitute only about $12 \%$ of the MACHO optical depth and only $4 \%$ of the EROS-2 optical depth.

The Galactic models are capable of reproducing the $t_{\mathrm{E}}$ distribution by adopting an appropriate stellar mass function. The model of Wood \& Mao (2005) is in good agreement with our distribution and with that of OGLE-II, both with $\left\langle t_{\mathrm{E}}\right\rangle \sim 28 \mathrm{~d}$. The model of Bissantz et al. (2004) is in good agreement with the distribution reported by MACHO with $\left\langle t_{\mathrm{E}}\right\rangle \sim 21 \mathrm{~d}$. According to Wood \& Mao (2005), the different $\left\langle t_{\mathrm{E}}\right\rangle$ predictions of the two models is mostly due to different adopted mass functions leading to a mean lensing mass of $0.35 M_{\odot}$ for Wood \& Mao (2005) and $0.11 M_{\odot}$ for Bissantz et al. (2004). Kinematical differences between the two models may also have a non-negligible effect.

The present comparison with models is still primarily limited by the low number of observed events. Since the EROS-2 survey uses most of the fields in the Bulge region that are easily observed in optical bands, a large increase in the number of monitored clump giants would require infrared observations of highly obscured regions near the Galactic Center (Gould 1995). Of course the number of events can also be increased by using dim stars but, in this case, reliable results would require a good understanding of blending.

Acknowledgements. We thank V. Belokurov and N.W. Evans for providing their microlensing maps in computer-readable form and O. Gerhard for interesting discussions. We are grateful to the technical staff of ESO, La Silla for the support given to the EROS-2 project. We thank J.-F. Lecointe and A. Gomes for the assistance with the online computing and the staff of the CC-IN2P3, especially the team in charge of the HPSS storage system, for their help with the data management. AG was supported by grant AST-0452758 from the NSF and JA by the Danish Natural Science Research Council.

\section{References}

Afonso, C., Albert, J. N., Andersen, J., et al. (EROS-2 collaboration) 2003a, A\&A, 400, 951

Afonso, Albert, J. N., Alard, C. C., et al. (EROS-2 collaboration) 2003b, A\&A, 404, 145

Alard, C. 1996, ApJ, 458, L17

Alcock, C., Allsman, R. A., Alves, D. R., et al. (MACHO collaboration) 1997, ApJ, 479, 119

Alcock, C., Allsman, R. A., Alves, D. R., et al. (MACHO collaboration) 2000a, ApJ, 541, 734

Alcock, C., Allsman, R. A., Alves, D. R., et al. (MACHO collaboration) 2000b, ApJ, 542, 281

Ansari, R. (EROS-2 collaboration) 1996, Vistas in Astronomy, 40, 519

Bauer, F., et al. (EROS-2 collaboration) 1997, Proceeding of the "Optical Detectors for Astronomy" workshop, ESO

Benjamin, R. A., Churchwell, E., Babler, B. L., et al. 2005, ApJ, 630, L149

Binney, J., Gerhard, O., \& Spergel, D. 1997, MNRAS, 388, 365

Binney, J., Bissantz, N., \& Gerhard, O. 2000, ApJ, 537, L99

Bissantz, N., \& Gerhard, O. 2002, MNRAS, 330, 591

Bissantz, N., Debattista, V., \& Gerhard, O. 2004, ApJ, 601, L155

Calchi Novati, S., Paulin-Henriksson, S., An, J., et al. 2005, A\&A, 443, 911

de Jong, J. T. A., Widrow, L. M., Cseresnjes, P., et al. 2005, A\&A, 446, 855

Derue, F., Afonso, C., Alard, C., et al. (EROS-2 collaboration) 2001, A\&A, 373, 126

Dwek, E., Arendt, R. G., Hauser, M. G., et al. 1995, ApJ, 445, 716

Evans, N. W., \& Belokurov, V. 2002, ApJ, 567, L119

Freudenreich, H. T. 1998, ApJ, 492, 495

Glicenstein, J. F. 2003, ApJ, 584, 278

Gould, A. 1995, ApJ, 446, L71

Griest, K. 1991, ApJ, 366, 412

Hamadache, C. 2004, Thesis, Université de Strasbourg,

http://tel.ccsd.cnrs.fr/

Han, C., \& Gould, A. 1995, ApJ, 449, 521

Han, C., \& Gould, A. 2003, ApJ, 592, 172

Holtzman, J. A., Watson, A. M., Baum, W. A., et al. 1998, AJ, 115, 1946

Joshi, Y. C., Pandey, A. K., Narasimha, D., \& Sagar, R. 2005, A\&A, 433, 787

Kiraga, M., \& Paczyński, B. 1994, ApJ, 430, L101 
Klypin, A., Zhao, H., \& Somerville, R. 2002, ApJ, 573, 597

Le Guillou, L. 2004, Thesis, Université de Paris VI,

http://tel.ccsd.cnrs.fr/

Paczyński, B. 1986, ApJ, 304, 1

Paczyński, B. 1991, ApJ, 371, L63

Popowski, P., Cook, K. H., Drake, A. J., et al. (MACHO collaboration) 2000, In Proc. Microlensing 2000, ed. J. W. Menzies, \& P. D. Sackett, ASP Conf. Ser. [arXiv: astro-ph/005466]

Popowski, P., Griest, K., Thomas, C. L., et al. 2005, ApJ, 631, 879

Riffeser, A., Fliri, J., Bender, R., et al. 2003, ApJ, 599, L17

Stanek, K. Z., Mateo, M., Udalski, A., et al. 1994, ApJ, 429, L73

Sumi, T., Abe, F., Bond, I. A., et al. (MOA collaboration) 2003, ApJ, 591, 204

Sumi, T., Wozniak, P. R., Udalski, A., et al. 2006, ApJ, 636, 240.
Tisserand, P., et al. 2006, in preparation; Tisserand, P. 2004, Thesis Université de Nice, http://tel.ccsd.cnrs.fr/

Udalski, A., Szymanski, M., Kaluzny, J., et al. (OGLE collaboration) 1994a, ApJ, 426, L69

Udalski, A., Szymanski, M., Stanek, K. Z., et al. (OGLE collaboration) 1994b, Act. Astr., 44, 165

Udalski, A., Zebrun, K., Szymanski, M., et al. (OGLE collaboration) 2000, Act. Astr., 50, 1

Udalski, A., Szymanski, M., Kubiak, M., et al. 2002, Act. Astr., 52, 217

Uglesich, R. R., Crotts, A. P. S., Baltz, E. A., et al. 2004, ApJ, 612, 877

Wood, A., \& Mao, S. 2005, MNRAS, 362, 945

Woźniak, P. R., Udalski, A., Szymanski, M., et al. (OGLE collaboration) 2001, Act. Astr., 51, 175 
C. Hamadache et al.: Microlensing optical depth towards the Galactic Bulge from EROS-2, Online Material p 1

\section{Online Material}


C. Hamadache et al.: Microlensing optical depth towards the Galactic Bulge from EROS-2, Online Material p 2

Table 3. The 120 events used for the measurement of the optical depth. The first column gives the identification: field, CCD, quarter, star number. The time of maximum amplification, $t_{0}$, is shown in the eighth column as (JD -2450000$)$. The efficiency, $\epsilon$, used in the optical depth calculation is shown in the ninth column. The final column signals events of special character: P (parallax), X (xallarap or binary lens without caustic), C (caustic binary lens), B (strong blend). Event numbers in the final column preceded by M refer to MACHO events (Popowski et al. 2005) and those preceded by SC refer to OGLE-II events Sumi et al. (2006).

\begin{tabular}{|c|c|c|c|c|c|c|c|c|c|}
\hline Event & RA & Dec & $b$ & $l$ & $t_{\mathrm{E}}$ & $u_{0}$ & $t_{0}$ & $\epsilon$ & Note \\
\hline $607-1-4-11480$ & 270.2603 & -29.0032 & -2.942 & 1.698 & 12.780 & 0.689 & 1274.70 & 0.576 & SC30-57488 \\
\hline 607-3-3- 9841 & 270.2787 & -29.3148 & -3.110 & 1.441 & 17.354 & 0.691 & 716.60 & 0.577 & M-113.19192.365 \\
\hline $607-5-1-14742$ & 270.0417 & -29.5630 & -3.053 & 1.121 & 18.181 & 0.067 & 2113.40 & 0.713 & \\
\hline $607-5-1-29607$ & 270.0288 & -29.6350 & -3.079 & 1.054 & 61.540 & 0.133 & 636.60 & 0.823 & M-118.18797.1397 \\
\hline $607-5-4-20285$ & 270.2924 & -29.8155 & -3.367 & 1.020 & 36.233 & 0.498 & 1315.50 & 0.810 & M-118.19184.939 \\
\hline $607-6-3-29248$ & 269.8908 & -30.0002 & -3.156 & 0.680 & 15.967 & 0.709 & 2137.00 & 0.560 & \\
\hline $610-0-1-17627$ & 270.4365 & -28.9678 & -3.059 & 1.809 & 18.775 & 0.347 & 1775.80 & 0.671 & SC30-636963 \\
\hline $610-2-3-4703$ & 270.6194 & -29.2331 & -3.328 & 1.666 & 10.458 & 0.420 & 1988.40 & 0.497 & \\
\hline 611-1-4- 24874 & 271.0400 & -27.7431 & -2.920 & 3.132 & 27.429 & 0.619 & 683.50 & 0.798 & M-104.20515.498 \\
\hline $611-3-4-18476$ & 271.1397 & -28.1256 & -3.184 & 2.849 & 13.708 & 0.699 & 998.40 & 0.706 & M-104.20640.8423 \\
\hline $612-7-4-8538$ & 271.8072 & -32.0593 & -5.593 & -0.195 & 70.213 & 0.233 & 1019.10 & 0.867 & \\
\hline $613-5-1-23714$ & 271.5512 & -30.1737 & -4.493 & 1.288 & 20.356 & 0.175 & 2144.10 & 0.722 & \\
\hline $615-1-2-22556$ & 271.8863 & -26.7402 & -3.090 & 4.378 & 14.728 & 0.126 & 1599.30 & 0.650 & \\
\hline 619-0-4- 7664 & 272.2137 & -28.4529 & -4.170 & 3.057 & 9.705 & 0.408 & 523.40 & 0.474 & M-110.22455.842 \\
\hline 619-5-4- 19755 & 272.7316 & -29.0649 & -4.862 & 2.769 & 16.290 & 0.507 & 1732.70 & 0.608 & \\
\hline 619-7-4- 14204 & 272.7335 & -29.4067 & -5.026 & 2.478 & 116.099 & 0.264 & 1254.70 & 0.677 & $\mathrm{P}$ \\
\hline $624-4-3-3703$ & 273.1066 & -30.1842 & -5.679 & 1.984 & 17.672 & 0.635 & 1414.90 & 0.588 & \\
\hline 627-7-2- 14994 & 273.4693 & -26.4997 & -4.217 & 5.306 & 43.505 & 0.041 & 1958.20 & 0.692 & \\
\hline 2-0-1- 13467 & 267.8171 & -30.2087 & -1.710 & -0.441 & 59.171 & 0.519 & 2383.30 & 0.793 & \\
\hline $2-0-4-18520$ & 268.1808 & -30.4511 & -2.103 & -0.482 & 22.388 & 0.239 & 1707.10 & 0.682 & \\
\hline 2-1-1- 5329 & 268.3954 & -30.1464 & -2.109 & -0.125 & 26.760 & 0.506 & 2122.60 & 0.783 & \\
\hline $2-3-3-30438$ & 268.4314 & -30.6361 & -2.383 & -0.524 & 38.098 & 0.552 & 996.30 & 0.908 & \\
\hline $2-5-1-20157$ & 268.2981 & -30.9266 & -2.432 & -0.831 & 97.853 & 0.088 & 1726.80 & 0.863 & $\mathrm{C}$ \\
\hline 3-4-3- 2992 & 268.1774 & -29.4347 & -1.585 & 0.381 & 65.395 & 0.476 & 2100.30 & 0.782 & \\
\hline 3-4-3- 27217 & 268.2445 & -29.5728 & -1.705 & 0.294 & 14.034 & 0.129 & 1685.60 & 0.582 & SC37-645044 \\
\hline 3-5-2- 20099 & 268.3380 & -29.6770 & -1.828 & 0.248 & 14.104 & 0.645 & 1759.70 & 0.554 & SC3-371229 \\
\hline $3-5-3-26926$ & 268.6611 & -29.5536 & -2.008 & 0.501 & 23.762 & 0.437 & 1257.30 & 0.706 & SC4-522952 \\
\hline $3-6-4-15890$ & 268.2890 & -30.0199 & -1.965 & -0.066 & 38.201 & 0.066 & 955.90 & 0.489 & SC3-91382 \\
\hline 4-3-2- 5389 & 269.2294 & -30.2536 & -2.786 & 0.164 & 16.954 & 0.527 & 1283.20 & 0.822 & \\
\hline $4-3-3-2508$ & 269.4168 & -30.0798 & -2.839 & 0.397 & 13.941 & 0.233 & 1411.40 & 0.753 & \\
\hline 4-4-4- 9143 & 268.9565 & -30.6300 & -2.770 & -0.280 & 21.513 & 0.044 & 1719.10 & 0.797 & B \\
\hline $4-5-2-8464$ & 269.0497 & -30.6251 & -2.837 & -0.233 & 17.209 & 0.456 & 1985.60 & 0.744 & \\
\hline 4-7-1- 7864 & 269.1321 & -30.8306 & -3.002 & -0.370 & 137.482 & 0.728 & 2086.20 & 0.930 & $P$ \\
\hline $4-7-1-20818$ & 269.1327 & -30.9075 & -3.041 & -0.435 & 14.483 & 0.614 & 2180.60 & 0.707 & \\
\hline 4-7-2- 19218 & 269.2468 & -31.0564 & -3.200 & -0.509 & 37.820 & 0.688 & 1833.10 & 0.912 & SC22-414328 \\
\hline 5-4-1- 8951 & 268.8382 & -29.0702 & -1.897 & 0.993 & 10.424 & 0.724 & 1093.70 & 0.537 & \\
\hline $5-4-3-22912$ & 269.0256 & -29.1386 & -2.073 & 1.020 & 30.119 & 0.492 & 2063.00 & 0.825 & \\
\hline 5-4-4- 11338 & 268.9376 & -29.2330 & -2.055 & 0.900 & 7.704 & 0.640 & 1271.10 & 0.411 & B \\
\hline 5-5-2- 19037 & 269.2080 & -29.2700 & -2.277 & 0.992 & 7.912 & 0.000 & 1651.60 & 0.411 & \\
\hline $6-1-3-21678$ & 270.0438 & -30.3994 & -3.468 & 0.411 & 13.550 & 0.060 & 1763.90 & 0.625 & $\mathrm{C}$ \\
\hline $6-5-3-18380$ & 270.1206 & -31.0448 & -3.844 & -0.102 & 23.286 & 0.208 & 2077.50 & 0.688 & \\
\hline $8-3-1-1345$ & 270.1013 & -27.7379 & -2.195 & 2.709 & 78.728 & 0.726 & 1714.00 & 0.907 & \\
\hline $8-4-2-21125$ & 269.7872 & -28.4151 & -2.290 & 1.986 & 34.932 & 0.358 & 948.70 & 0.801 & X M-401.48408.649 \\
\hline $8-4-2-21623$ & 269.6680 & -28.3593 & -2.171 & 1.979 & 43.148 & 0.124 & 2129.90 & 0.887 & \\
\hline $8-7-3-16227$ & 270.2820 & -28.5282 & -2.724 & 2.114 & 19.994 & 0.469 & 1301.90 & 0.803 & SC30-165305 \\
\hline 9-7-4- 7143 & 271.0161 & -31.5437 & -4.755 & -0.118 & 50.982 & 0.510 & 1450.80 & 0.914 & \\
\hline $30-2-3-19447$ & 273.9211 & -28.5940 & -5.557 & 3.712 & 23.998 & 0.398 & 1698.10 & 0.758 & \\
\hline $31-3-3-35856$ & 274.4120 & -27.2411 & -5.308 & 5.095 & 13.824 & 0.318 & 1628.90 & 0.503 & \\
\hline $61-0-3-8256$ & 274.4475 & -22.4102 & -3.067 & 9.292 & 37.373 & 0.654 & 1743.70 & 0.781 & \\
\hline $70-3-1-18517$ & 265.4297 & -34.2263 & -2.068 & -4.914 & 90.589 & 0.181 & 1707.40 & 0.964 & $\mathrm{C}$ \\
\hline $70-3-1-18797$ & 265.3838 & -34.2285 & -2.036 & -4.936 & 91.418 & 0.207 & 2400.50 & 0.961 & \\
\hline $70-3-4-28566$ & 265.5989 & -34.4414 & -2.300 & -5.016 & 29.865 & 0.425 & 1602.40 & 0.871 & \\
\hline $70-4-1-17316$ & 265.0553 & -34.5743 & -1.989 & -5.374 & 7.140 & 0.567 & 1428.90 & 0.330 & \\
\hline $70-5-2-24247$ & 265.3672 & -34.7616 & -2.306 & -5.388 & 71.090 & 0.061 & 1842.60 & 0.890 & \\
\hline 70-6-2- 31993 & 264.9205 & -35.1415 & -2.197 & -5.908 & 46.872 & 0.246 & 1456.10 & 0.587 & \\
\hline
\end{tabular}


C. Hamadache et al.: Microlensing optical depth towards the Galactic Bulge from EROS-2, Online Material p 3

Table 3. continued.

\begin{tabular}{|c|c|c|c|c|c|c|c|c|c|}
\hline Event & RA & Dec & $b$ & $l$ & $t_{\mathrm{E}}$ & $u_{0}$ & $t_{0}$ & $\epsilon$ & Note \\
\hline $71-3-1-21364$ & 266.2818 & -33.3554 & -2.214 & -3.797 & 47.100 & 0.073 & 892.10 & 0.911 & \\
\hline $71-7-1-28518$ & 266.4633 & -34.0936 & -2.727 & -4.332 & 36.263 & 0.177 & 2024.70 & 0.906 & $\mathrm{C}$ \\
\hline $72-1-1-22000$ & 266.3057 & -34.4337 & -2.792 & -4.688 & 18.317 & 0.131 & 2343.10 & 0.722 & \\
\hline $72-4-2-12551$ & 265.8097 & -35.1914 & -2.841 & -5.544 & 21.568 & 0.461 & 1442.30 & 0.804 & \\
\hline 73-3-4- 7001 & 267.3455 & -33.4813 & -3.040 & -3.418 & 14.286 & 0.043 & 896.30 & 0.739 & \\
\hline $74-5-3-16791$ & 267.4200 & -35.0934 & -3.917 & -4.730 & 23.496 & 0.180 & 2177.00 & 0.793 & \\
\hline $76-1-3-12658$ & 268.3605 & -31.6522 & -2.845 & -1.417 & 82.468 & 0.676 & 1046.30 & 0.900 & \\
\hline $77-0-1-20761$ & 267.5647 & -33.1794 & -3.042 & -3.065 & 18.932 & 0.487 & 928.60 & 0.711 & \\
\hline $77-0-3-7242$ & 267.7949 & -33.0623 & -3.149 & -2.862 & 128.844 & 0.151 & 1628.20 & 0.883 & $\mathrm{P}$ \\
\hline 77-1-4- 10694 & 268.3326 & -33.2374 & -3.625 & -2.765 & 24.832 & 0.419 & 2005.60 & 0.853 & \\
\hline 77-4-1- 13517 & 267.7165 & -33.8108 & -3.473 & -3.525 & 23.493 & 0.144 & 1426.00 & 0.856 & \\
\hline $78-4-1-3363$ & 268.7127 & -31.8944 & -3.225 & -1.461 & 8.176 & 0.430 & 1387.10 & 0.492 & \\
\hline $79-2-1-8900$ & 268.4667 & -33.0348 & -3.620 & -2.534 & 29.763 & 0.344 & 1680.00 & 0.832 & \\
\hline 80-3-2- 7178 & 269.8741 & -32.2297 & -4.246 & -1.216 & 35.887 & 0.049 & 1047.00 & 0.876 & \\
\hline $80-4-2-10880$ & 269.4209 & -32.6063 & -4.100 & -1.740 & 10.348 & 0.733 & 2040.10 & 0.548 & \\
\hline $81-3-2-24224$ & 269.7945 & -33.7675 & -4.946 & -2.545 & 30.017 & 0.253 & 1341.00 & 0.887 & \\
\hline $82-2-3-24164$ & 270.3955 & -32.2755 & -4.652 & -1.018 & 13.832 & 0.285 & 2357.80 & 0.631 & \\
\hline $83-1-1-8206$ & 270.5674 & -33.2471 & -5.253 & -1.758 & 13.159 & 0.180 & 1315.10 & 0.614 & \\
\hline $84-4-1-15036$ & 271.1088 & -33.0547 & -5.556 & -1.351 & 19.032 & 0.000 & 2176.00 & 0.727 & \\
\hline $101-1-1-20136$ & 263.7159 & -29.3984 & 1.743 & -1.622 & 10.623 & 0.094 & 2351.60 & 0.292 & B \\
\hline $102-0-3-17623$ & 262.5237 & -29.4664 & 2.577 & -2.223 & 25.501 & 0.152 & 1322.90 & 0.593 & \\
\hline $102-3-1-13451$ & 262.6484 & -29.7646 & 2.322 & -2.420 & 12.616 & 0.290 & 1093.50 & 0.554 & \\
\hline $102-5-2-15625$ & 262.7204 & -30.2758 & 1.990 & -2.822 & 24.927 & 0.139 & 2197.60 & 0.587 & \\
\hline $102-5-3-29920$ & 262.9454 & -30.2184 & 1.858 & -2.671 & 33.810 & 0.586 & 1348.90 & 0.642 & \\
\hline $103-1-4-21106$ & 263.1299 & -28.1925 & 2.829 & -0.858 & 23.528 & 0.310 & 2380.90 & 0.630 & \\
\hline $103-3-4-27325$ & 263.1901 & -28.5500 & 2.590 & -1.137 & 10.030 & 0.662 & 1777.20 & 0.463 & \\
\hline $104-3-3-29860$ & 262.2052 & -29.1929 & 2.959 & -2.134 & 12.518 & 0.142 & 2064.20 & 0.572 & \\
\hline $104-3-4-27383$ & 262.1975 & -29.3197 & 2.895 & -2.246 & 20.467 & 0.575 & 1984.30 & 0.651 & \\
\hline $105-5-1-14483$ & 261.1343 & -29.5197 & 3.553 & -2.900 & 7.628 & 0.682 & 1636.30 & 0.334 & \\
\hline $105-7-4-12278$ & 261.2801 & -30.0158 & 3.171 & -3.256 & 25.769 & 0.356 & 1604.50 & 0.739 & \\
\hline 106-1-4- 11177 & 265.1363 & -27.0417 & 1.947 & 1.043 & 30.726 & 0.324 & 1624.90 & 0.565 & \\
\hline $106-4-1-8809$ & 264.7175 & -27.5637 & 1.985 & 0.404 & 5.775 & 0.298 & 1701.50 & 0.291 & \\
\hline $106-4-4-24841$ & 264.8552 & -27.8152 & 1.748 & 0.251 & 24.738 & 0.453 & 1073.50 & 0.785 & B \\
\hline $106-4-4-31028$ & 264.8404 & -27.8541 & 1.739 & 0.211 & 17.878 & 0.535 & 1623.80 & 0.687 & \\
\hline $106-5-1-15482$ & 265.0711 & -27.6027 & 1.699 & 0.531 & 19.899 & 0.122 & 1293.10 & 0.610 & \\
\hline $106-5-1-26077$ & 264.9651 & -27.6713 & 1.742 & 0.424 & 11.974 & 0.593 & 2099.10 & 0.462 & \\
\hline $106-5-4-24760$ & 265.1471 & -27.8188 & 1.527 & 0.381 & 25.477 & 0.093 & 2524.00 & 0.679 & \\
\hline $106-6-1-13061$ & 264.6275 & -27.9500 & 1.847 & 0.032 & 36.059 & 0.283 & 1687.00 & 0.508 & $\mathrm{C}$ \\
\hline $107-0-2-21213$ & 263.4739 & -26.7017 & 3.382 & 0.578 & 124.365 & 0.025 & 1250.10 & 0.776 & \\
\hline $107-1-2-24707$ & 263.7571 & -26.7073 & 3.166 & 0.702 & 16.090 & 0.404 & 2080.40 & 0.543 & \\
\hline $107-3-3-31150$ & 263.9854 & -26.9224 & 2.878 & 0.621 & 19.904 & 0.591 & 1309.50 & 0.754 & \\
\hline $107-4-1-8762$ & 263.3894 & -27.1358 & 3.210 & 0.166 & 35.398 & 0.489 & 1754.90 & 0.758 & \\
\hline $107-4-4-23806$ & 263.7192 & -27.3873 & 2.827 & 0.100 & 53.574 & 0.650 & 1055.00 & 0.881 & \\
\hline $107-5-2-26345$ & 263.8999 & -27.3882 & 2.692 & 0.182 & 14.064 & 0.197 & 1710.70 & 0.576 & \\
\hline $107-7-1-22583$ & 263.8056 & -27.5884 & 2.654 & -0.033 & 8.574 & 0.075 & 1635.90 & 0.415 & B \\
\hline 107-7-2- 167 & 263.9081 & -27.6208 & 2.561 & -0.014 & 9.466 & 0.220 & 1616.40 & 0.455 & \\
\hline $108-3-1-12859$ & 266.2558 & -25.8209 & 1.736 & 2.604 & 12.864 & 0.499 & 1658.60 & 0.585 & \\
\hline $108-3-1-27549$ & 266.2520 & -25.9237 & 1.685 & 2.513 & 11.375 & 0.197 & 888.20 & 0.504 & \\
\hline $108-4-1-13544$ & 265.9802 & -26.1798 & 1.760 & 2.169 & 53.105 & 0.689 & 1322.70 & 0.854 & \\
\hline $108-4-1-23818$ & 265.7825 & -26.2489 & 1.874 & 2.019 & 99.504 & 0.672 & 2532.60 & 0.823 & \\
\hline $109-4-3-15519$ & 265.2998 & -25.5322 & 2.621 & 2.417 & 17.836 & 0.417 & 1365.00 & 0.686 & \\
\hline $112-0-2-12627$ & 266.7715 & -23.3296 & 2.629 & 4.995 & 21.280 & 0.615 & 1317.10 & 0.639 & \\
\hline $112-2-2-25953$ & 266.8246 & -23.7485 & 2.371 & 4.655 & 20.012 & 0.700 & 1648.60 & 0.765 & \\
\hline $112-5-2-24472$ & 267.1992 & -24.0774 & 1.907 & 4.541 & 23.119 & 0.676 & 1011.60 & 0.558 & \\
\hline $112-6-1-4305$ & 266.7831 & -24.1610 & 2.190 & 4.279 & 10.871 & 0.293 & 2473.80 & 0.363 & \\
\hline $112-7-3-14923$ & 267.3655 & -24.2420 & 1.693 & 4.474 & 54.797 & 0.612 & 1813.50 & 0.789 & \\
\hline $113-1-2-28239$ & 266.3130 & -23.4511 & 2.926 & 4.681 & 23.803 & 0.066 & 1967.50 & 0.768 & \\
\hline $113-7-1-23134$ & 266.3956 & -24.3175 & 2.411 & 3.967 & 26.167 & 0.294 & & & \\
\hline 863.60 & 0.817 & & & & & & & & \\
\hline
\end{tabular}


C. Hamadache et al.: Microlensing optical depth towards the Galactic Bulge from EROS-2, Online Material p 4

Table 3. continued.

\begin{tabular}{llllllllll}
\hline \hline Event & RA & Dec & $b$ & $l$ & $t_{\mathrm{E}}$ & $u_{0}$ & $t_{0}$ & $\epsilon$ & Note \\
\hline $114-3-1-11753$ & 265.5659 & -23.5507 & 3.458 & 4.254 & 18.387 & 0.076 & 2044.50 & 0.728 & \\
$114-5-1-16265$ & 265.5462 & -23.9263 & 3.276 & 3.919 & 67.940 & 0.548 & 1664.30 & 0.810 & $\mathrm{X}$ \\
$117-6-3-13250$ & 268.0484 & -22.5049 & 2.042 & 6.293 & 6.270 & 0.082 & 1643.30 & 0.203 & \\
$117-7-1-12630$ & 268.2245 & -22.5131 & 1.898 & 6.366 & 24.160 & 0.100 & 1794.70 & 0.761 & \\
$124-1-1-16687$ & 259.9039 & -27.8925 & 5.362 & -2.066 & 19.173 & 0.180 & 1410.20 & 0.688 & \\
$124-7-2-20832$ & 259.9118 & -29.1101 & 4.664 & -3.106 & 201.882 & 0.311 & 2075.30 & 0.854 & $\mathrm{P}$ \\
$124-7-3-21344$ & 260.0548 & -28.9748 & 4.638 & -2.925 & 47.000 & 0.295 & 918.50 & 0.851 & \\
\hline
\end{tabular}

